\title{
Investigations of the Optical Spectroscopy of Atomic Sodium Isolated in Solid Argon and Krypton: Experiments and Simulations. ${ }^{\dagger}$
}

\author{
Maryanne Ryan, $\stackrel{\ddagger}{\ddagger}$ Martin Collier, $\stackrel{\ddagger}{ }$ Patrick de Pujo, ${ }^{\S}$ Claudine Crépin, ${ }^{*, \perp}$ and \\ John G. McCaffrey \\ Department of Chemistry, National University of Ireland - Maynooth, Maynooth, County Kildare, Ireland, \\ Laboratoire Francis Perrin, CEA/DSM/IRAMIS/SPAM CNRS URA 2453, CEA Saclay, 91191 Gif-sur-Yvette, \\ France, and Laboratoire de PhotoPhysique Moléculaire, CNRS UPR 3361, Bât. 210- Université Paris-Sud 11, \\ F91405 Orsay Cedex, France
}

Received: June 15, 2009; Revised Manuscript Received: August 10, 2009

The absorption spectra of thin film samples, formed by the codeposition of sodium vapor with the rare gases have long been known to consist of complex structures in the region of the atomic sodium "yellow-doublet" lines. The photophysical characteristics of the associated luminescence (excitation/emission) spectra, indicate strong interaction between the excited $\mathrm{P}$ state $\mathrm{Na}$ atom and the rare gases $(\mathrm{Ar}, \mathrm{Kr}$, and $\mathrm{Xe}$ ) used as host solids. This system is reinvestigated with new experimental spectroscopic results and molecular dynamics (MD) calculations. The so-called "violet" site in Ar and $\mathrm{Kr}$ has been produced by laser excitation of thermally deposited samples. The simulation of the "spray-on" deposition of thin films enables identification of tetravacancy (tv) sites of isolation for ground-state atomic sodium in Ar while in $\mathrm{Kr}$ this site is found in addition to single vacancy (sv) occupancy. Various cubic symmetry sites were taken into account to simulate absorption and emission spectra using accurate interaction potentials for the $\mathrm{Na} \cdot \mathrm{RG}$ diatomics. The wellknown 3-fold splitting in absorption, attributed to the Jahn-Teller effect, was very well reproduced but the simulated spectra for all the sites considered are located in the low energy region of the experimental bands. The evolution of the excited state $\mathrm{Na}$ atom is followed revealing the nature and symmetry of the sites that are transiently occupied. Consistent with the large Stokes shift observed experimentally, there is an extensive rearrangement of the lattice in the excited state with respect to the ground state. Combining all the experimental and theoretical information, an assignment of experimental violet, blue, and red absorption features is established involving single vacancy, tetravacancy, and hexavacancy sites, respectively, in Ar and $\mathrm{Kr}$.

\section{Introduction}

The spectroscopy of matrix isolated sodium $(\mathrm{Na} / \mathrm{RG})$ has been extensively examined over the past 30 years. ${ }^{1-5}$ Initial interest in this system arose from the spectroscopic simplicity of the gas phase atomic sodium ${ }^{2} \mathrm{P}-{ }^{2} \mathrm{~S}$ transition but even in the earliest investigations, several complexities were noted in the absorption spectra of the $\mathrm{Na} / \mathrm{RG}$ matrix systems. Chief among these is the unusual behavior observed on deposition, that the absorption bands do not show the expected red shift on going from the light to the heavy hosts (Ar to Xe). Thus the atomic ${ }^{2} \mathrm{P} \leftarrow{ }^{2} \mathrm{~S}$ transition spans the range 16000 to $19000 \mathrm{~cm}^{-1}$ in the spectra recorded for all three host matrices $-\mathrm{Ar}, \mathrm{Kr}$, and $\mathrm{Xe}$. Another peculiar characteristic is that in annealed samples, the thermally stable site in $\mathrm{Na} / \mathrm{Xe}$ absorbs at higher energy than what appear to be the thermally stable sites in Ar and Kr. Yet another unusual observation, made by Tam and Fajardo ${ }^{3}$ for samples prepared by the co-condensation of laser ablated sodium atoms with the rare gases $\mathrm{Ar}$ and $\mathrm{Kr}$, is the existence of another site of isolation. Fajardo referred to these features as the violet sites as they were located at higher energy than any bands observed in samples produced with thermal vaporization of sodium.

\footnotetext{
"Part of the "Benoît Soep Festschrift".

* To whom correspondence should be addressed. E-mail: claudine.crepingilbert@u-psud.fr .

* National University of Ireland, Maynooth.

$\S$ CEA/DSM/IRAMIS/SPAM CNRS URA 2453.

${ }^{\perp}$ CNRS UPR 3361.
}

To date, these peculiarities remain largely unanswered despite numerous experimental studies and more recent theoretical investigations. ${ }^{6-9}$ Despite the considerable body of experimental and theoretical data that exists for the $\mathrm{Na} \cdot \mathrm{RG}$ diatomics, determination of the trapping sites of the metal atom in raregas matrices in particular remains an open question due to the large differences between the single-vacancy (sv) site sizes of the solid rare-gases and the $\mathrm{Na} \cdot \mathrm{RG}$ bond lengths. For instance the $\mathrm{Na} \cdot \mathrm{Ar}$ bond length is, as indicated in Table 1, larger than a single vacancy site in $\mathrm{Ar}$ by $1.21 \AA$. Even in $\mathrm{Xe}$, the discrepancy is still considerable being $0.62 \AA$. A key objective of the present study is to identify the trapping sites of the metal atom in each matrix. The cases of solid argon and krypton show many similarities and will be more specifically explored in this paper.

In 1978, Balling et al. ${ }^{1}$ recorded absorption and emission spectra of $\mathrm{Na}$ atoms isolated in solid argon (Ar), krypton ( $\mathrm{Kr}$ ), and xenon (Xe) matrices. Four distinct absorption bands, labeled $\mathrm{A}, \mathrm{B}, \mathrm{C}$, and $\mathrm{D}$, in order of increasing energy, were observed

TABLE 1: Lattice Parameter (a), Site Sizes for the Solid Rare-Gas Host in a fec Unit Cell, ${ }^{47}$ and $\mathrm{Na} \cdot \mathrm{RG}$ Ground-State Bond Lengths. All Distances Are Quoted in Angstroms (ঐ)

\begin{tabular}{lclcccc}
\hline $\mathrm{RG}$ & $a$ & \multicolumn{1}{c}{$\mathrm{I}_{\mathrm{Oh}}$} & $\mathrm{sv}$ & $\mathrm{tv}$ & $\mathrm{hv}$ & $\mathrm{Na} \cdot \mathrm{RG}$ \\
\hline $\mathrm{Ar}$ & 5.31 & 2.655 & 3.755 & 4.403 & 4.598 & 5.01 \\
$\mathrm{Kr}$ & 5.64 & 2.82 & 3.988 & 4.676 & 4.884 & 4.92 \\
$\mathrm{Xe}$ & 6.13 & 3.065 & 4.330 & 5.083 & 5.308 & 4.95
\end{tabular}


for $\mathrm{Na}$ atoms isolated in argon. Features $\mathrm{A}$ and $\mathrm{B}$, which henceforth shall be referred to as red and blue sites, respectively, exhibited triplet structure. The peaks for the blue site appear at approximately 554,545 , and $536 \mathrm{~nm}$, while those for the red site are at 594, 587, and $577 \mathrm{~nm}$. Three sites were observed in the Kr system and were labeled A, B, and D, also in order of increasing energy. Triplet features for the red site in krypton occur at 606,595 , and $578 \mathrm{~nm}$ with the blue site absorbing at 576,569 , and $560 \mathrm{~nm}$. Luminescence spectra of $\mathrm{Na} / \mathrm{Ar}$ were recorded using a pulsed dye-laser tuned to the absorption bands of both sites. Broad emission bands, centered at approximately 705 and $770 \mathrm{~nm}$, were recorded for the red site in argon while blue site emission bands in the same matrix were at 675 and $755 \mathrm{~nm}$. Excitation of the blue and red sites in $\mathrm{Kr}$ revealed emission bands at $720 \mathrm{~nm}$ for the red site; however, two maxima at 705 and $820 \mathrm{~nm}$ were observed for blue site emission. Balling and Wright ${ }^{2}$ also performed calculations to simulate the formation of the trapping site of $\mathrm{Na}$ in argon and xenon. They proposed that the blue sites in both matrices correspond to the isolation of the $\mathrm{Na}$ atom in a single vacancy site, while the red site, which is thermally unstable, results from "amorphous sites evolving from two-atom vacancies".

In 1993, Tam and Fajardo ${ }^{3}$ carried out new experimental work on the $\mathrm{Na} / \mathrm{RG}$ systems by generating sodium vapor with laser ablation. Both red and blue triplet absorptions were recorded along with a higher-energy feature referred to as the "violet" site. This new site was also reported by Silverman and Fajardo in experiments involving deposition of $\mathrm{Na}^{+}$ions in Ar matrices. Fajardo and co-workers ${ }^{3,8}$ assigned the highest energy absorption to the smallest volume trapping site; however, specific identification of the trapping site was not made. Later Boatz and Fajardo ${ }^{9}$ carried out Monte Carlo simulations of the site structures and optical absorption spectra of $\mathrm{Na}$ atoms in $\mathrm{Ar}$ clusters, surfaces, and solids. Various trapping sites were considered and those structures with cubic $\left(\mathrm{O}_{\mathrm{h}}\right.$ or $\left.\mathrm{T}_{\mathrm{d}}\right)$ symmetry generated the triplet absorption band shape observed in the experimental work. ${ }^{1,2}$ A theoretical study of atomic sodium in argon clusters and matrices has already been made by Gro $\beta$ and Spiegelman ${ }^{10}$ using a pseudopotential method. For a Na atom isolated in a substitutional site of an fcc (face centered cubic) Ar lattice containing 63 atoms, the results of the pseudopotential calculations found a strong blueshifted absorption at $471 \mathrm{~nm}$. This predicted band is clearly much higher in energy than the violet site which is observed at $510 \mathrm{~nm}$.

The origin of the triplet structure present on the absorption bands has been the subject of much debate in the past few decades. ${ }^{11}$ The principal question being whether crystal field effects, multiple site ${ }^{12}$ occupancy, or the Jahn-Teller ${ }^{13,14}$ (JT) effect are responsible for the structure. In their magnetic circular dichroïsm (MCD) study of the ${ }^{2} \mathrm{P} \leftarrow{ }^{2} \mathrm{~S}$ transition of $\mathrm{Na}$ and $\mathrm{Li}$ atoms in solid xenon, Schatz et al. ${ }^{4}$ presented compelling evidence 20 years ago that the JT effect produces the observed splitting.

In an attempt to resolve some of the unusual characteristics, we have undertaken a combined experimental and computational study of the spectroscopy of matrix isolated sodium atoms. The current work is focused on the isolation of atomic sodium in solid argon and krypton matrices. Experimentally sample preparation was achieved with two techniques involving both thermal vaporization and electron-bombardment. Absorption and luminescence spectra are obtained using both continuous lamp and pulsed laser excitation sources in the visible spectral region. In addition laser irradiation has been used very effectively to induce site interconversion. Using time-resolved emission spectroscopy, the fluorescence lifetimes of the isolated atoms in specific sites are obtained. Molecular dynamics (MD) calculations have also been undertaken to identify the sites of isolation on deposition and probe the excited state dynamics following optical excitation of the ${ }^{2} \mathrm{P}$ state. They are employed to simulate both the deposition of metal atoms in rare-gas matrices and in conjunction with the diatomics-in-molecule ${ }^{15}$ (DIM) method, to predict the electronic spectra of the resultant matrix-isolated atom. The use of a "simple" DIM method is preferred as a first approach to the problem in spite of the complexity of the interaction of the excited sodium atom with a rare gas surrounding. ${ }^{16,17}$ Accurate ground and excited state potential energy curves extracted from spectroscopic data for all the $\mathrm{Na} \cdot \mathrm{RG}$ diatomics are currently available, and our simulations are also a test of their validity in the matrix context. On the basis of these $\mathrm{Na} \cdot \mathrm{RG}$ pair-potentials, the MD calculations permit us to follow the internal motion of the trapped species within the lattice of the host matrix in both the ground and excited states. The motivation behind the simulation of a deposition is to identify the sites of isolation of the $\mathrm{Na}$ atom within the matrix.

A study, involving ab initio theoretical calculations, is currently underway on $\mathrm{Na} / \mathrm{Xe}$, the results of which will be presented in a future publication.

\section{Experimental Methods}

The experimental apparatus used in the preparation of matrixisolated sodium samples has been described in previous publications from the Maynooth group ${ }^{18,19}$ so only a summary is provided here. Matrices were prepared by co-condensing $\mathrm{Na}$ vapor, produced by both electron bombardment of $\mathrm{Na}$ metal in a Mo crucible, and by resistive heating. The isolation of $\mathrm{Na}$ atoms in the Ar matrix proved to be difficult with the former method so the oven source was used predominantly with this system. In this arrangement a tantalum Knusden cell was used to vaporize sodium and the rate was monitored with a quartz crystal microbalance. Solid $\mathrm{Na} / \mathrm{RG}(\mathrm{RG}=\mathrm{Ar}, \mathrm{Kr}$, and $\mathrm{Xe})$ samples were prepared by co-condensing $\mathrm{Na}$ vapor with the rare gas of interest onto a $\mathrm{CaF}_{2}$ window. The minimum temperature of $10.5 \mathrm{~K}$, achieved with an APD Cryogenics closed-cycle helium displex system (model HC-2), was, unless otherwise stated, used for the spectral scans reported and depositions. This temperature was achieved using a radiation shield and employed to produce samples as free as possible of sodium dimers and clusters.

Transmittance spectra were obtained with a Hamamatsu R928 photomultiplier tube (PMT) by scanning a $0.3 \mathrm{~m}$ Acton Research Corporation (ARC) SpectraPro(SP)-300i monochromator. Emission was recorded with a $0.5 \mathrm{~m}$ ARC SP-500i monochromator fitted with a $600 \mathrm{~g} / \mathrm{mm}$ grating blazed at $500 \mathrm{~nm}$. A Hamamatsu R928-P PMT, maintained at $-20{ }^{\circ} \mathrm{C}$ in a Products for Research (model S600) cooled-housing, was used in photon counting mode for emission detection. Excitation spectra were recorded with a tungsten lamp and the SP300i monochromator. A pulsed dye laser (Quantel, TDL-90) pumped with the second harmonic $(532 \mathrm{~nm})$ of a Nd:YAG (Quantel, YG989E-10) laser, operating at a repetition rate of $10 \mathrm{~Hz}$, was used for recording timeresolved luminescence. Laser excitation of the blue site was achieved using Rhodamine 590, while Rhodamine and DCM were used for red site excitation. Typical laser fluences of 20 $\mu \mathrm{J} / \mathrm{mm}^{2}$ in the visible beam were generated using only the oscillator of the TDL-90 dye laser. With pulsed laser excitation the emitted light was dispersed with the ARC SP-500i monochromator and detected with an intensified-gated, charged- 
TABLE 2: Spectroscopic Data from References ${ }^{22-25}$ Were Used to Generate the Potential Energy Curves of the $X$ and A States of the Diatomic Molecules $\mathrm{Na} \cdot \mathrm{Ar}$ and $\mathrm{Na} \cdot \mathrm{Kr}^{a}$

\begin{tabular}{|c|c|c|c|c|}
\hline & $\mathrm{X}^{2} \Sigma$ & $\mathrm{A}^{2} \Pi$ & $\mathrm{B}^{2} \Sigma$ & $\mathrm{B}^{2} \Sigma$ mod. \\
\hline \multicolumn{5}{|c|}{$\mathrm{Na} \cdot \mathrm{Ar}$} \\
\hline$R_{0}(\AA)$ & 4.996 & 2.903 & 6.759 & 6.974 \\
\hline$a_{6}$ & -44.5467967291 & -7089.546277 & 110.1510972888 & -139.04449800 \\
\hline$a_{10}$ & 1223.8558817715 & -27224.381688 & 1619.2809458919 & -35.65071706 \\
\hline$a_{12}$ & -901.07449919 & 22797.577245 & -1172.7772808718 & 4.13354921 \\
\hline$a_{14}$ & 342.2852434367 & -9355.030711 & 460.7939594123 & -0.23406619 \\
\hline$a_{20}$ & -0.3579059458 & & -0.6147206546 & \\
\hline \multicolumn{5}{|c|}{$\mathrm{Na} \cdot \mathrm{Kr}$} \\
\hline$R_{0}(\AA)$ & 4.998 & 3.077 & 6.414 & 6.412 \\
\hline$a_{6}$ & -118.1934163466 & -6657.947390 & -12.2587131762 & -233.39074724 \\
\hline$a_{8}$ & -979.4078458243 & 10824.888220 & -841.9158775356 & 546.50469493 \\
\hline$a_{10}$ & 2222.3797768480 & -9537.867307 & 1513.9419747217 & -1853.04520496 \\
\hline$a_{20}$ & -1.2408829460 & & -0.9798801319 & 16.58993304 \\
\hline$a_{22}$ & & & 0.0398447503 & \\
\hline$a_{24}$ & & & -0.0005426194 & \\
\hline
\end{tabular}

${ }^{a}$ The data for the $\mathrm{B}$ states of $\mathrm{Na} \cdot \mathrm{Ar}$ are theoretical results from ref 30 , while the $\mathrm{Na} \cdot \mathrm{Kr}$ data are experimental data from ref 32 . Using eq 2 and a standard least-squares fit the $a_{n}$ coefficients presented in the table were extracted from these data, the results of which are shown in Figure 1. All coefficients are quoted in Kelvin. The coefficients indicated as "B ${ }^{2} \Sigma$ mod." represent the more repulsive B state potentials shown by the dashed line in Figure 1 and used in the matrix spectral simulations.

coupled device (iCCD) camera (Andor Technologies, model iStar DH720). Both time-integrated and nanosecond timeresolved emission spectra (TRES) have been obtained with this device using pulsed laser excitation. To extract fluorescent lifetimes, decay curves were obtained at specific emission wavelengths by taking kinetic slices of the TRES. To verify that true radiative lifetimes are being identified, TRES were recorded for preannealed samples and measurements were made over a range of temperatures starting at $12.7 \mathrm{~K}, 16 \mathrm{~K}$ and then increasing in steps of four degrees up to $34 \mathrm{~K}$. Lifetimes were extracted by fitting single or multiple exponential functions to the recorded decay curves with the linear, least-squares method.

\section{Theoretical Methods}

The principal aim of the theoretical study is to identify the trapping sites of sodium atoms in solid argon and krypton matrices. Therefore, modeling the experimental deposition process is a key part of the study as it assists our understanding of how the guest atom is accommodated in its solid state surrounding.

III.A. Potentials. All the MD calculations undertaken in the present study are based on pair potentials. The $\mathrm{RG}_{2}$ potentials were modeled on the Lennard-Jones function with $\sigma$ values of 3.3605 and $3.58 \AA$ used for $\mathrm{Ar}^{20}$ and $\mathrm{Kr}^{21}$ respectively, and $\epsilon$ values of 142.1 and $199.2 \mathrm{~K}$. These values were obtained from a variety of experimental data on solid and gaseous $\mathrm{Ar}$ and $\mathrm{Kr}$. Atomic sodium, with the electronic configuration $[\mathrm{Ne}] 3 \mathrm{~s}^{1}$, has $\mathrm{a}^{2} \mathrm{~S}_{1 / 2}$ ground state and first excited ${ }^{2} \mathrm{P}_{1 / 2}$ and ${ }^{2} \mathrm{P}_{3 / 2}$ states, derived from the $[\mathrm{Ne}] 3 \mathrm{p}^{1}$ configuration. The latter states produce the strongly bound $\mathrm{A}{ }^{2} \Pi$ and repulsive $\mathrm{B}{ }^{2} \Sigma$ state when in the presence of a rare gas atom, while the ground state sodium yields the weakly bound $\mathrm{X}^{2} \Sigma$ state. Interatomic potential energies for the $\mathrm{X}$ and $\mathrm{A}$ states for both $\mathrm{Na} \cdot \mathrm{Ar}$ and $\mathrm{Na} \cdot \mathrm{Kr}$ were obtained from the experimental work of Schwarzhans et al. ${ }^{22,23}$ and Zimmermann and co-workers, ${ }^{24,25}$ respectively, who employed a tunable dye laser as the excitation source for the diatomic $\mathrm{Na} \cdot \mathrm{RG}$ molecules stabilized in a low temperature supersonic jet. The interatomic potentials were then extracted from the spectroscopic data and fit with analytical functions such as Tang-Toennies, ${ }^{26}$ Thakkar, ${ }^{27}$ or Hartree-Fock-dispersion (HFD) ${ }^{28}$ The HFD-function was employed to describe both the $\mathrm{X}$ and $\mathrm{A}$ states for diatomic $\mathrm{Na} \cdot \mathrm{Ar}-$ the parameters of these states are listed in Tables 5.2 and 5.5 of ref 23 . Both the $A^{2} \Pi_{1 / 2}$ and $A^{2} \Pi_{3 / 2}$ states were utilized in the present simulations. However, because of the small splitting between these two states, we shall only present the results of the calculations undertaken using data for the lower energy $\mathrm{A}^{2} \Pi_{1 / 2}$ state. For $\mathrm{Na} \cdot \mathrm{Kr},{ }^{24} \mathrm{a}$ modified $\mathrm{HFD}^{28}$ function was used to describe the $\mathrm{X}$ state, while the Morse function was utilized for the A state. The B state used in the simulations of the absorption and emission spectra of the $\mathrm{Na} \cdot \mathrm{Ar}$ system were those calculated by Kerner and Meyer ${ }^{29,30}$ These theoretical results were in good agreement with experimental results reported by Grosser, Hoffmann, and Rebentrost ${ }^{31}$ for the repulsive B potential. Rebentrost and coworkers ${ }^{32}$ determined the $\mathrm{Na} \cdot \mathrm{Kr}, \mathrm{B}^{2} \Sigma_{1 / 2}$ state potential from collision-induced absorption data. The data given in their work cover the range from 3.40 to $5.55 \AA$.

The data sets for the ground and excited state potentials were then fit to the analytical function given by eq 1 for use in the MD programs. The $a_{2(k+2)}$ coefficients, determined by fitting the $\mathrm{X}, \mathrm{A}$ and $\mathrm{B}$ states are collected in Table 2.

$$
V(R)=\sum_{k=1}^{n} a_{2(k+2)}\left(\frac{R_{0}}{R}\right)^{2(k+2)}
$$

Plots depicting the potential energy curves of the three electronic states for both diatomic molecules $\mathrm{Na} \cdot \mathrm{Ar}$ and $\mathrm{Na} \cdot \mathrm{Kr}$, are presented in Figure 1. For the purposes of comparing the shapes of the three potentials, each curve in this plot is shown 


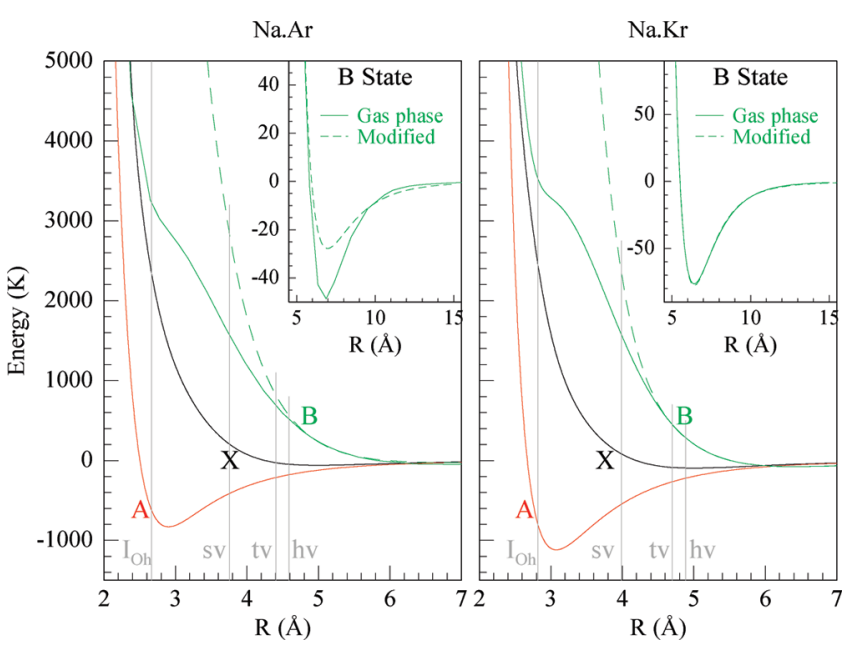

Figure 1. Interaction potentials of diatomic $\mathrm{Na} \cdot \mathrm{Ar}$ and $\mathrm{Na} \cdot \mathrm{Kr}$ molecules for the three states utilized in this study. The A and B states arise from the interaction of the excited ${ }^{2} \mathrm{P}$ state of sodium with the rare gas atom while the $\mathrm{X}$ state is the ground ${ }^{2} \mathrm{~S}$ state interaction. The bound $\mathrm{X}$ and $\mathrm{A}$ curves were obtained using Zimmermann's HFD functions ${ }^{23}$ (discussed in the text) while the $\mathrm{B}$ state of $\mathrm{Na} \cdot \mathrm{Ar}$ was obtained from the work of Kerner and Meyer. ${ }^{29,30}$ In the case of $\mathrm{Na} \cdot \mathrm{Kr},{ }^{24}$ the HFD function was also used to produce the X state of $\mathrm{Na} \cdot \mathrm{Kr}$, while the A state was found using the Morse function. Collisioninduced optical data available from the work of Rebentrost et al. was employed for the B state. ${ }^{32}$ Also shown are the nearest neighbor distances in the three most important sites of the solid $\mathrm{Ar}$ and $\mathrm{Kr}$ hosts for the isolation of a spherical guest, viz, single vacancy (sv), tetravacancy (tv), and hexavacancy (hv). The octahedral interstitial $\left(\mathrm{I}_{\mathrm{Oh}}\right)$ site size is shown for comparison. The numerical values for each of these sites are collected in Table 1. Modifications made to the B state are indicated by the dashed line. This modification increases the repulsive part at short distances and shown in the inset, weakens the attractive part around $7 \AA$ in Ar.

with a common asymptotic limit of zero. In reality, the A and B states dissociate to the ${ }^{2} \mathrm{P}$ asymptote, $16956 \mathrm{~cm}^{-1}$ above the ground X state. Also shown in these plots, by the vertical lines, are the sizes (listed in Table 1) of the key sites in the solid rare gases considered capable of accommodating a spherical guest.

III.B. Deposition. MD calculations were devised to simulate the Na/RG matrix formation. Simulating the growth of a sample, formed by the spray-on of the metal vapor and rare gas onto the cold window, is achieved by creating projectiles that collide with a surface generated prior to the MD run. Crépin et al..$^{33}$ presented details of these simulated depositions in an earlier paper. The simulations were achieved using molecular dynamics (MD) calculations developed and conducted at the CEA, Saclay.

Briefly, deposition commences on a prefabricated square slab consisting of four layers of rare-gas atoms constructed in the (100) conformation of the fcc lattice and parallel to the $x y$-plane of a space-fixed axis system. The dimensions of the four prefabricated layers are $45 \times 45 \times 9 \AA^{3}{ }^{3}$ The first layer is fixed in space in order to prevent rotational and translational motion of the lattice during the deposition procedure. The second layer functions as a thermostat. During deposition, the temperature is maintained by rescaling the velocities of all the atoms in the second layer. ${ }^{34}$ In the third and fourth layers, atoms are free to move within the applied interaction forces. The translational differential equations are solved using the Verlet-velocity algorithm. ${ }^{35}$ Deposition of individual samples required a maximum of $60 \mathrm{~ns}$ with an integration step $(\delta t)$ of 5 fs. Typically 400-600 atoms, including one sodium atom, are deposited and all computations were undertaken at temperatures between 5 and $55 \mathrm{~K}$.
Both fast and slow depositions were carried out with time intervals ranging from 20 to 100 ps between the arrival of adatoms. This is sufficient time to allow the lattice to relax between incoming atoms. Experimental deposition corresponds to the arrival of approximately one atom every $40 \mu \mathrm{s}$; however, the MD calculations do not have the capacity to cover this time range. Fortuitously, it is found that the most important factor in designing calculations that reproduce experimental conditions is the lattice relaxation time and not the deposition rate. The lattice relaxation time has been estimated as $100 \mathrm{ps}$ in the simulations. Thus, the slower deposition is more realistic with respect to the experimental conditions. RG adatoms may impact any location on the 'prefabricated' surface, however, the impact of the $\mathrm{Na}$ atom is directed to the middle of the $x y$-plane. The initial velocity of the adatoms was taken as the mean thermal velocity of the rare gases at room temperature. In all the MD calculations, a cutoff distance of $15 \AA$ is applied to eliminate surface effects while reducing the computation time.

III.C. Model Sites. Model matrices of the rare gas solids were created, containing nine layers of the face-centered cubic (fcc) lattice. Each matrix cube, with dimensions $40 \AA \times 40 \AA$ $\times 40 \AA$, contained a total of 1293 atoms and the layers were constructed according to the (111) conformation. To this cube a sodium atom was placed in a well-defined site. The purpose of building the cubes with model sites is to examine the full range of possible sites occupied by atomic sodium in the simulated depositions. In particular, we examined the case of cubic site symmetry with the sodium atom placed in sv, $t v, \mathrm{Io}_{\mathrm{h}}$, or hv sites. These regular structure sites were chosen because of their high symmetry since the experimental absorption spectra have consistently exhibited 3 -fold splitting. This effect has been attributed to JT coupling with lattice modes-an effect ${ }^{14}$ occurring in sites of high symmetry such as exists when the guest atom is in sv (cuboctahedral), tv (tetrahedral), $\mathrm{Io}_{\mathrm{h}}$ or hv (octahedral) sites. The $\mathrm{Io}_{\mathrm{h}}$ site is a vacancy intrinsic to the close packed fcc lattice. It corresponds to the void between six atoms arranged as a triangle on one (111) layer and an oppositely oriented triangle from an adjacent layer. A tv site is created with the removal of a tetrahedron of four adjacent host atoms surrounding a tetrahedral interstitial $\left(\mathrm{I}_{\mathrm{Td}}\right)$ site in the fcc lattice. When the six atoms (described above) surrounding an $\mathrm{Io}_{\mathrm{h}}$ site are removed, a hexavacancy is produced. Pure rare-gas lattices were also generated for the purpose of comparison with the $\mathrm{Na}-$ doped lattices.

Analysis of the structure of the sites accommodating the sodium atom was made by determining the $\mathrm{Na}$ atom-rare gas atom pair distances. These results are presented in the form of eq 2, the radial pair-distribution function $G(r)$ defined as

$$
G(r)=\frac{h(r)}{N_{\mathrm{c}} 4 \pi r^{2} \Delta r}
$$

In this expression $h(r)$ is the histogram of the pair distances between $\mathrm{Na}$ and rare gas atoms at a particular distance $r, \Delta r$ is the step width used in the calculations (fixed at $0.05 \AA$ in this work) while $N_{\mathrm{c}}$ is the number of configurations included in the averaging. In pure RG matrices, $G(r)$ is obtained with a rare gas atom replacing $\mathrm{Na}$ for the sv site, while pair distances are calculated from a point at the center of $\mathrm{I}_{\mathrm{Td}}$ or $\mathrm{Io}_{\mathrm{h}}$ interstitial sites for the other sites. Comparison of these functions for each model matrix site with those obtained in the simulated depositions enables direct identification of the site of isolation of the sodium atom. In addition, the thermal stabilities of the model 
sites at various temperatures were examined as a further aid in site identification.

III.D. Simulating Absorption Spectra. Simulations of the absorption spectra were achieved using the diatomics-inmolecule (DIM) method. ${ }^{15}$ The Hamiltonians for both ground and excited state surfaces are constructed according to the method outlined in the work of Visticot et al. ${ }^{36}$ This method follows the classical evolution of the system in the ground state, whereby the distribution of nuclear configurations of the atoms on the ground state potential energy surface is established. The energies of the total Hamiltonian in the excited and ground states are calculated for all these configurations. Diagonalization of the matrix follows and the three resultant transition energies are retained. The energy histogram obtained at the end of the computation corresponds to the absorption spectrum.

At the cryogenic temperatures of the experimental work, the zero-point energy of the lattice is nearly equal to the thermal energy. Therefore, in classical calculations, temperature scaling is required to bring the results closer to quantum reality. The thermal harmonic quantum correction ${ }^{37}$ adjusts the temperature of the calculations and in so doing, takes into account the vibrational amplitudes of the rare-gas atoms. The effective temperature, $T^{\prime}$, is found by matching the classical probability distribution for a harmonic oscillator to the quantum probability distribution $^{37}$ in the limit goes to zero and is given by eq 3 .

$$
T^{\prime}=\frac{\hbar \omega}{2 k_{\mathrm{B}}}\left(\tanh \left(\frac{\hbar \omega}{2 k_{\mathrm{B}} T}\right)\right)^{-1}
$$

In this equation, $T^{\prime}$ is the scaled temperature, $T$ is the experimental temperature, $k_{\mathrm{B}}$ is Boltzmann's constant, and $\omega$ is the angular frequency of the nuclear motion. The effective temperature was found to be important in reproducing the bandwidths of the absorption and emission spectra. In this study, all absorption and emission spectra in the Ar matrix are carried out at $45 \mathrm{~K}$ while those of $\mathrm{Kr}$ are carried out at $35 \mathrm{~K}$. Both correspond to an experimental temperature of around $12 \mathrm{~K}$.

III.E. Simulating Emission Spectra. Simulation of the emission spectra must of course be done with MD calculations on the excited state. However, it is known that the excited ${ }^{2} \mathrm{P}$ state of the metal atom in the matrix is described by three potential energy curves, labeled $\mathrm{E}_{1}, \mathrm{E}_{2}$, and $\mathrm{E}_{3}$. To avoid computationally demanding calculations on several potential surfaces, as Tully ${ }^{38}$ has done with the molecular dynamics with quantum transitions (MDQT) method, we have used the previous results of Rojas-Lorenzo et al. ${ }^{39}$ on the $\mathrm{Hg} / \mathrm{Ar}$ system to develop an efficient method for determining the emission spectra. Using Tully's MDQT method, Rojas-Lorenzo et al. showed a rapid depopulation (several picoseconds) from the two highest energy excited states to the excited state of lowest energy due to many crossings between the three states. The energies of the three excited states of the $\mathrm{Na} / \mathrm{RG}$ system have also been followed as a function of displacements occurring on the ground state. The diabatic curves obtained are illustrated in Figure 2 for the $\mathrm{Na} /$ Ar system over a time interval of $10 \mathrm{ps}$. As observed with the $\mathrm{Hg} / \mathrm{Ar}$ system, many crossings occur between the three excited states in the $\mathrm{Na} / \mathrm{Ar}$ system. We conclude that, as in the $\mathrm{Hg} / \mathrm{Ar}$ system, only the lowest excited surface is populated after a relaxation time of a few picoseconds. Moreover, following this excited surface, the two higher excited surfaces lie at a high energy level (more than $2000 \mathrm{~cm}^{-1}$ above the lowest excited surface). Accordingly, in a determination of the emission spectra, it is reasonable to conduct the molecular dynamics calculation on just the lowest energy excited state potential surface.

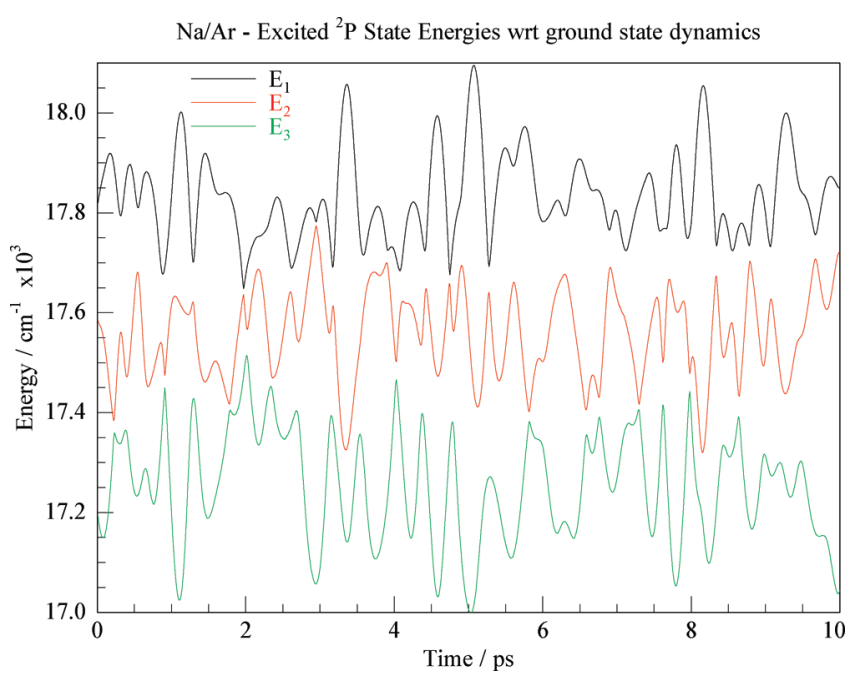

Figure 2. Energies of the three states, derived from the free atom excited ${ }^{2} \mathrm{P}$ state, versus time during a MD run on the ground electronic state. Multiple crossings are evident between the potential surfaces indicating rapid relaxation to the lowest energy state.

Thus at each step of the MD calculation, the energy of the lowest excited state and its derivatives with respect to each of the coordinates of the system are evaluated using the HellmannFeynman theorem applied to $[\mathbf{H}]$ - a symmetrical, real $3 \times 3$ matrix associated with the Hamiltonian $\mathbf{H}$. If $\mathbf{a}$ is a real parameter, such as a nuclear coordinate and $\mathbf{X}$ is the eigenvector associated to the eigenvalue $\mathbf{E}$, then we have

$$
\frac{\mathrm{d} \mathbf{E}(\mathbf{a})}{\mathrm{d} \mathbf{a}}=\mathbf{X}^{\mathrm{T}} \frac{\mathrm{d} \mathbf{H}(\mathbf{a})}{\mathrm{d} \mathbf{a}} \mathbf{X}
$$

In this manner the lowest energy level, $\mathrm{E}_{3}$, is located and the trajectory is mapped out along the classical evolution of the system in this excited state. When the stabilized configuration is identified on level $\mathrm{E}_{3}$, the emission spectrum is constructed by calculating the energy difference between this state and the ground state, along the classical evolution of the system in this excited state.

\section{Results: Experimental}

IV.A. Absorption Spectra. The visible absorption spectra recorded with a tungsten lamp yielded bands in good agreement with the published results of Balling et al. ${ }^{1}$ The spectra of freshly deposited matrices, presented on the left panel in Figure 3, show multiple 3-fold split bands in the vicinity of the gas phase $3 p$ ${ }^{2} \mathrm{P} \leftrightarrow 3 \mathrm{~s}{ }^{2} \mathrm{~S}$ transition of atomic sodium at $589.75 \mathrm{~nm}$. In solid Ar, two distinct trapping environments are suggested by the 3 -fold split bands centered at 543.5 and $585.2 \mathrm{~nm}$. Historically these sites are referred to as the "blue" and "red" sites of atomic sodium in solid Ar with the red site dominating the spectrum by nearly a factor of 3 . However, the red site is found to be thermally unstable. In $\mathrm{Kr}$ and $\mathrm{Xe}$ the blue and red sites are partially overlapped but are centered at 565.9/593.9 and 560.8/ $603.2 \mathrm{~nm}$, respectively. Recording excitation spectra provided clearer identification of the sites in $\mathrm{Kr}$ and $\mathrm{Xe}$. These are shown on the left in Figure 4. As observed in Ar, the red site is also less thermally stable than the blue site in these heavier hosts.

The changes occurring in the freshly deposited $\mathrm{Na} / \mathrm{RG}$ samples following pulsed-laser excitation of the $\mathrm{Na}^{2} \mathrm{P}$ state are shown on the right in Figure 3. Clearly a higher energy, 3-fold split absorption band has been produced in $\mathrm{Na} / \mathrm{Ar}$ and $\mathrm{Na} / \mathrm{Kr}$ 


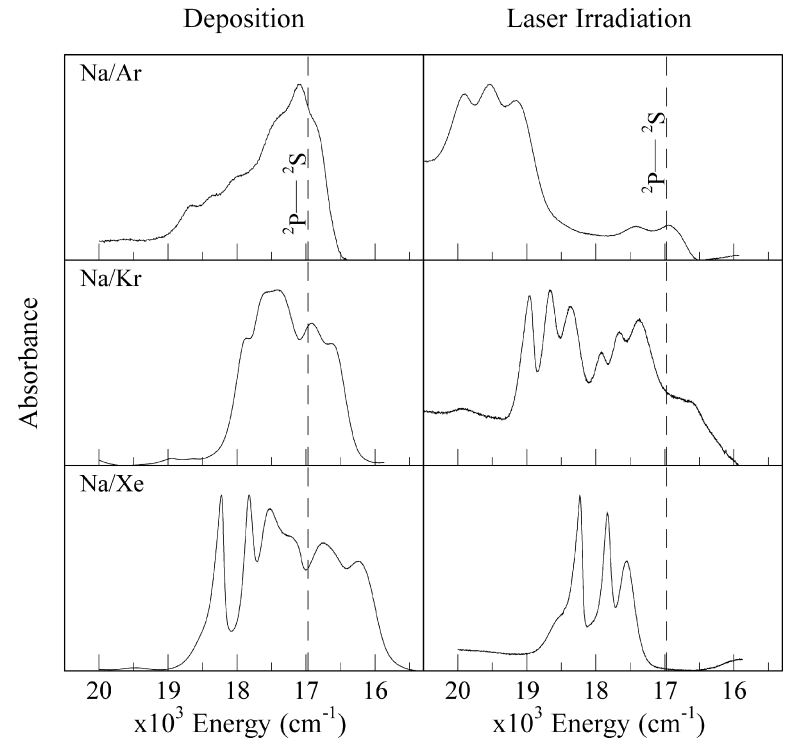

Figure 3. The absorption spectra recorded on deposition at $10 \mathrm{~K}$ in the vicinity of the atomic $\mathrm{Na}^{2} \mathrm{P} \leftarrow{ }^{2} \mathrm{~S}$ transition for the $\mathrm{Na} / \mathrm{Ar}, \mathrm{Na} / \mathrm{Kr}$, and $\mathrm{Na} / \mathrm{Xe}$ systems are shown in the panel on the left. An indication of the change induced in these samples with laser irradiation at 557, 595 , and $595 \mathrm{~nm}$ for $\mathrm{Na} / \mathrm{Ar}, \mathrm{Na} / \mathrm{Kr}$, and $\mathrm{Na} / \mathrm{Xe}$, respectively, is given by comparison with the right-hand panel. The emergence of the highest energy absorption, referred to as the "violet" site, is evident in the case of the $\mathrm{Na} / \mathrm{Ar}$ and $\mathrm{Na} / \mathrm{Kr}$ systems, while removal of the red site is the only change induced in the $\mathrm{Na} / \mathrm{Xe}$ system.

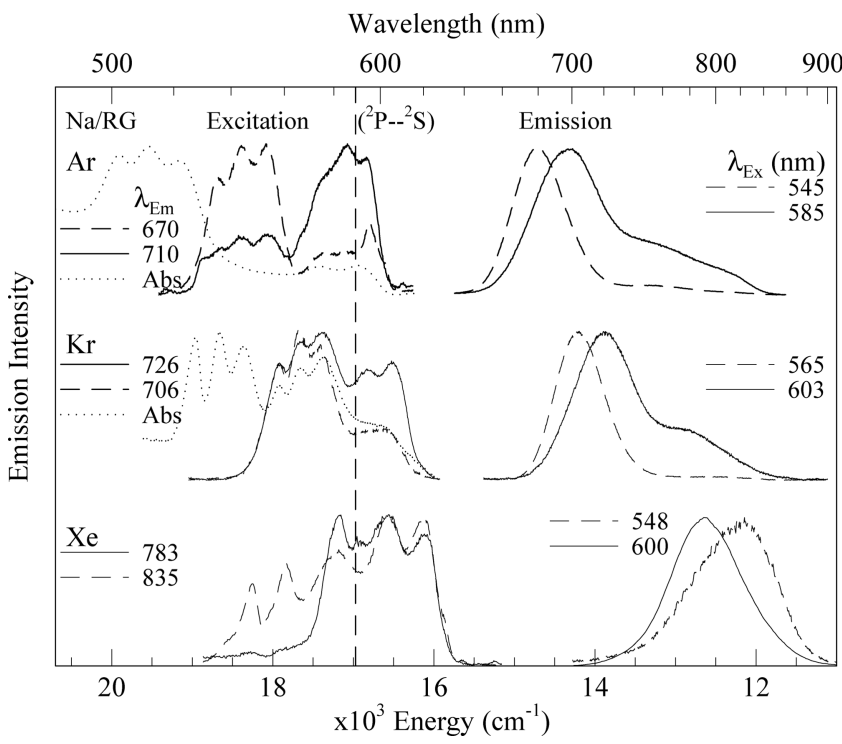

Figure 4. Luminescence spectra recorded for $\mathrm{Na} / \mathrm{Ar}, \mathrm{Na} / \mathrm{Kr}$, and $\mathrm{Na} /$ Xe with lamp excitation of the atomic absorptions shown in the previous figure. The emission in all three systems consists of two well-defined bands and a red wing in the $\mathrm{Ar}$ and $\mathrm{Kr}$ systems. The excitation spectra, presented on the left of the figure reveal that only the red and blue sites produce emission. The profiles shown by the dotted trace for the violet site in $\mathrm{Ar}$ and $\mathrm{Kr}$ are absorption spectra. It is noteworthy that the lower energy emission in $\mathrm{Ar}$ and $\mathrm{Kr}$ are produced with red site excitation, while in Xe the behavior is different-the lowest energy emission is produced with blue site excitation.

with this treatment while no new features are evident in $\mathrm{Na} /$ $\mathrm{Xe}$. The red site has diminished in all samples following laser irradiation. The new bands produced in $\mathrm{Ar}$ and $\mathrm{Kr}$ correspond to the high-energy "violet" trapping sites already reported by Tam and Fajardo ${ }^{3}$ for matrix-isolated atomic sodium produced by laser ablation.

IV.B. Luminescence. Excitation and emission spectra of $\mathrm{Na}$ isolated in a solid argon matrix at $10 \mathrm{~K}$ and annealed to $29 \mathrm{~K}$ are presented in the upper section of Figure 4. The emission spectrum recorded with blue-site excitation at $545 \mathrm{~nm}$ is centered at $679 \mathrm{~nm}$. 3-Fold splitting is evident in the excitation spectrum recorded monitoring this emission band at $670 \mathrm{~nm}$ with resolved features at 535,545 , and $552 \mathrm{~nm}$. Red site excitation at 585 $\mathrm{nm}$ produces emission centered at $698 \mathrm{~nm}$ with a red wing extending out to $830 \mathrm{~nm}$. It is noteworthy that the red site in Ar produces the lowest energy emission while blue excitation produces the highest energy emission. Details of the luminescence and Stokes' shift are collected in Table 3. The red site gives rise to a Stokes shift of $2760 \mathrm{~cm}^{-1}$, while it is $3670 \mathrm{~cm}^{-1}$ for the blue site. In contrast to the red and blue sites, the violet band did not produce emission in the region 500-850 $\mathrm{nm}$. The band shown for the violet site (the dotted trace on the upper left in Figure 4) is an absorption profile.

The luminescence spectroscopy of atomic sodium isolated in a krypton matrix is presented in the middle portion of Figure 4. Two distinct emission bands, shown on the right of the figure, are observed with intensity maxima at 703.8 and $719.7 \mathrm{~nm}$. The emission band observed at $704 \mathrm{~nm}$ is produced with excitation of the blue site. The emission centered at $720 \mathrm{~nm}$ results from red site excitation at 581, 593, or $602 \mathrm{~nm}$. Large Stokes shifts are observed for both sites with average values listed in Table 3 as 2943 and $3462 \mathrm{~cm}^{-1}$ for the red and blue sites, respectively. It is clear in Figure 4, that the $\mathrm{Na} / \mathrm{Kr}$ emission bears a strong resemblance to that of $\mathrm{Na} / \mathrm{Ar}$ with the blue site producing the highest energy emission while the red site produces the lowest energy emission and a red wing extending to $840 \mathrm{~nm}$. As found for $\mathrm{Na} / \mathrm{Ar}$, the violet site in $\mathrm{Na} / \mathrm{Kr}$ did not yield emission.

$\mathrm{Na} / \mathrm{Xe}$ produced the simplest emission with just two bands. As shown in the bottom of Figure 4 one emission band is centered at $790 \mathrm{~nm}$, the other is at $821 \mathrm{~nm}$. The shape of the latter band, produced with blue site excitation at $548 \mathrm{~nm}$, is asymmetrical as it extends beyond the limit of our detection system at $850 \mathrm{~nm}$. This band was observed to be weak in all the samples prepared even though it emanates from excitation of the thermally stable site centered at $560.8 \mathrm{~nm}$. The higher energy $790 \mathrm{~nm}$ emission is produced with the red site excitation at $600 \mathrm{~nm}$ while the lower energy emission at $821 \mathrm{~nm}$ is produced with blue site excitation. Thus the behavior in $\mathrm{Na} / \mathrm{Xe}$ is different to that observed in the $\mathrm{Na} / \mathrm{Ar}$ and $\mathrm{Na} / \mathrm{Kr}$ systems in which the lowest energy absorption produced the lowest energy emission.

IV.C. Time Resolved Emission. Time-resolved emission spectra (TRES) produced with pulsed laser excitation and $\mathrm{iCCD}$ detection were recorded, and decay profiles were extracted from the TRES as described in section II. Fitting the decay curves with single exponential functions allowed us to determine the lifetimes $\tau$ of all the observed emission bands for the three matrix systems. Measurements made as a function of temperature indicated that the values recorded at $10 \mathrm{~K}$ are the radiative lifetimes. These values are collected in column 6 of Table 3 and are plotted in Figure 5 as well as a $\lambda^{3}$ extrapolation of the $16.8 \mathrm{~ns}$ gas phase lifetime. The directly observed matrix values are all less than the gas phase extrapolation. However, when the observed matrix lifetimes, $\tau_{\mathrm{obs}}$, are corrected with Fulton's expression $^{40}$

$$
\tau_{\text {cor }}=\tau_{\text {obs }} n\left(\frac{n^{2}+2}{3}\right)^{2}
$$

for the effective field of the solids, only the two sites in Ar fall on the $\lambda^{3}$ curve. In this expression $n$ is the index of refraction-values of $1.32,1.428$, and 1.49 have been used for 
TABLE 3: Spectral Positions of Atomic Spectroscopic Features Assigned for Atomic Sodium Isolated in Solid Ar and Kr on Deposition at $12.7 \mathrm{~K}^{a}$

\begin{tabular}{|c|c|c|c|c|c|}
\hline system & $\lambda_{\mathrm{ex}}\left(\mathrm{nm} / \mathrm{cm}^{-1}\right)$ & $\lambda_{\mathrm{em}}\left(\mathrm{nm} / \mathrm{cm}^{-1}\right)$ & $\mathrm{SS}\left(\mathrm{cm}^{-1}\right)$ & $\delta\left(\mathrm{cm}^{-1}\right)$ & $\tau(\mathrm{ns})$ \\
\hline gas phase & $589.75 / 16956$ & $589.75 / 16956$ & & & 16.9 \\
\hline \multicolumn{6}{|c|}{$\mathrm{Na} / \mathrm{Ar}$} \\
\hline \multirow{2}{*}{ violet } & $511.8 / 19539$ & NA & NA & +2583 & NA \\
\hline & $521.9 / 19161$ & & & & \\
\hline \multirow[t]{2}{*}{ blue } & $535.0 / 18692$ & & & & \\
\hline & $543.5 / 18399$ & $679 / 14728$ & 3671 & +1443 & 13.5 \\
\hline \multirow{2}{*}{ red } & $585.2 / 17088$ & $698 / 14327$ & 2761 & +132 & 13.5 \\
\hline & $593.9 / 16838$ & & & & \\
\hline \multicolumn{6}{|c|}{$\mathrm{Na} / \mathrm{Kr}$} \\
\hline \multirow[t]{2}{*}{ violet } & $527.4 / 18961$ & & & & \\
\hline & $535.8 / 18663$ & NA & NA & +1707 & NA \\
\hline \multirow{2}{*}{ red } & $593.9 / 16838$ & $719.7 / 13895$ & 2943 & -118 & 15.12 \\
\hline & $605.3 / 16521$ & & & & \\
\hline \multicolumn{6}{|c|}{$\mathrm{Na} / \mathrm{Xe}$} \\
\hline \multirow[t]{3}{*}{ blue } & $548.5 / 18231$ & & & & \\
\hline & $560.8 / 17832$ & $821.0 / 12180$ & 5652 & +875 & 28 \\
\hline & $569.6 / 17556$ & & & & \\
\hline \multirow[t]{2}{*}{ red } & $582.0 / 17182$ & & & & \\
\hline & $603.2 / 16578$ & $791.3 / 12637$ & 3940 & -378 & 22 \\
\hline
\end{tabular}

${ }^{a}$ The triplet peak positions are listed in column 2, the emission maxima for each site are listed in column 3, and the Stokes' shift (SS) are given in column 4 . The gas phase transition energies for the $\mathrm{Na}$ atom are also presented. In column five, the gas-to-matrix shift, denoted by $\delta$, is also listed. The $\mathrm{Na} / \mathrm{Kr}$ red site feature presented in brackets is overlapped by the blue site, its value is obtained in a line-shape analysis. Lifetimes are reported in column 6.

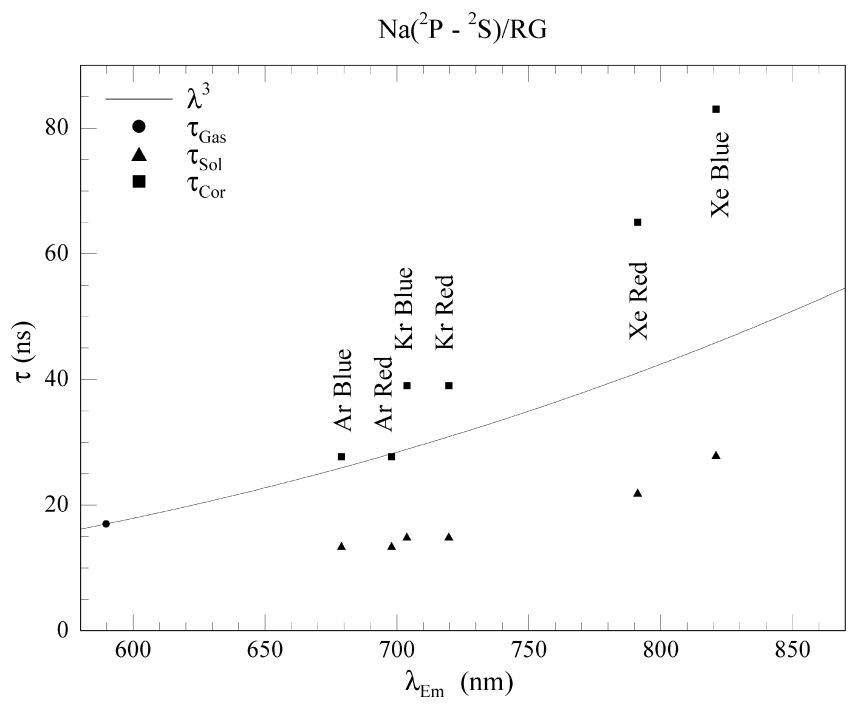

Figure 5. A comparison of the $\lambda^{3}$ dependence plot of the emission lifetime (16.8 ns) for the gas phase ${ }^{2} \mathrm{P}-{ }^{2} \mathrm{~S}$ transition of atomic $\mathrm{Na}$ and the observed and corrected emission lifetimes for the site specific emissions in $\mathrm{Na} / \mathrm{Ar}, \mathrm{Na} / \mathrm{Kr}$, and $\mathrm{Na} / \mathrm{Xe}$ matrices. The effective field correction made in the solids is given by eq 5 .

$\mathrm{Ar}, \mathrm{Kr}$, and $\mathrm{Xe}$, respectively. Both sites in $\mathrm{Kr}$ exhibit corrected lifetimes that are $50 \%$ longer than the extrapolated gas phase value. In Xe the deviation is on the order of $100 \%$. This behavior would indicate that the ${ }^{2} \mathrm{P}-{ }^{2} \mathrm{~S}$ transition is strongly perturbed especially in the heavier rare gases.

IV.D. Spectral Shifts. The experimental results on the $\mathrm{Na} /$ $\mathrm{Ar}$ and $\mathrm{Na} / \mathrm{Kr}$ systems reveal three sites of isolation, two of which were observed in the earliest works ${ }^{1}$ on matrix-isolated atomic sodium and one, the violet site, was found in the most recent works. ${ }^{3,8}$ Comparing, in Figure 3 the appearance of the 3 -fold splitting of the violet absorption band in $\mathrm{Na} / \mathrm{Kr}$ and the blue site in $\mathrm{Na} / \mathrm{Xe}$, it is evident that the two bands bear a strong resemblance. Moreover when the matrix shifts $\delta$, (data given in column 5 of Table 3 ) of the two violet bands and the blue site in Xe are plotted in Figure 6 against the polarizabilities of the hosts, it appears that a linear dependence exits. This behavior suggests that the same site is responsible for the violet sites in $\mathrm{Ar}$ and $\mathrm{Kr}$ and the blue site in $\mathrm{Xe}$, even though they are produced by very different means. When the data on the blue sites in $\mathrm{Ar}$ and $\mathrm{Kr}$ are plotted in a similar way it would appear they are best correlated with the red site in Xe.

Large Stokes shifts ranging between 2800 and $3900 \mathrm{~cm}^{-1}$ are observed for the emission of all sites in $\mathrm{Ar}, \mathrm{Kr}$, and $\mathrm{Xe}$ with the exception of the blue site in Xe which is much larger having a value of $5652 \mathrm{~cm}^{-1}$. It is noteworthy that the violet sites in $\mathrm{Ar}$ and $\mathrm{Kr}$, do not exhibit emission. Thus if this emission exists it is very weak or, if present, is beyond the lower energy limit of our detection range. These findings once again indicate that the $\mathrm{Na}$ atom is interacting especially strongly with the surrounding matrix when isolated in the violet site.

\section{Results: Theoretical}

V.A. Trapping Sites. "Model" matrices of the fcc lattice were created, as described in section III.C, for both $\mathrm{Ar}$ and $\mathrm{Kr}$ each containing nine layers in the (111) conformation. One of the models consisted entirely of rare gas atoms, that is, the pure 


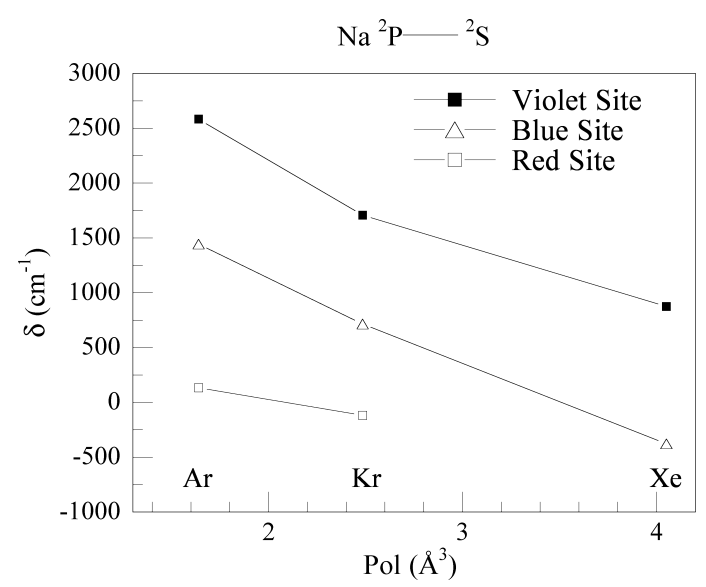

Figure 6. A plot of the gas phase to $\mathrm{Na} / \mathrm{RG}$ matrix frequency shifts $\left(\delta \mathrm{cm}^{-1}\right)$ observed for the violet, blue, and red sites identified for the ${ }^{2} \mathrm{P} \leftarrow{ }^{2} \mathrm{~S}$ transition versus the $\mathrm{RG}$ host polarizabilities. Gas-phase absorption ${ }^{46}$ for the ${ }^{2} \mathrm{P}_{1 / 2}-{ }^{2} \mathrm{~S}_{1 / 2}$ and ${ }^{2} \mathrm{P}_{3 / 2}-{ }^{2} \mathrm{~S}_{1 / 2}$ transitions of atomic $\mathrm{Na}$ occurs at $589.755 \mathrm{~nm}\left(16956.172 \mathrm{~cm}^{-1}\right)$ and $589.16 \mathrm{~nm}(16973.368$ $\mathrm{cm}^{-1}$ ) respectively. The latter value was used and the matrix values are presented in column 5 of Table 3 . The legend shown in the figure for the bands present in Xe differs from the labels used in the literature. Thus in the present analysis the violet sites in $\mathrm{Ar}$ and $\mathrm{Kr}$ correlate with the blue site in $\mathrm{Xe}$ while the blue sites in $\mathrm{Ar}$ and $\mathrm{Kr}$ correlate with the red site in $\mathrm{Xe}$.

rare gas lattice. In the remaining cubes, a $\mathrm{Na}$ atom was placed in various trapping sites either found or expected, viz, sv, tv, or a hv site.

Figure 7 presents the radial distribution functions calculated with eq 2 for both $\mathrm{Na} / \mathrm{Ar}$ (upper panels) and $\mathrm{Na} / \mathrm{Kr}$ (lower panels). In the top left plot, the radial distributions of the $\mathrm{Na}$ atom located in a tv site of the argon matrix is compared with that of a pure argon matrix calculated from the center of a tetrahedral interstitial $\left(\mathrm{I}_{\mathrm{Td}}\right)$ site. Ignoring the first peak, which of course is absent in the tv site, the extent of the overlap of the peaks implies that the $\mathrm{Na}$ atom occupying the tv site produces no distortion of the Ar lattice. In contrast, the top center panel of Figure 7 indicates the extent of the distortion of the matrix when a $\mathrm{Na}$ atom is "forced" into a sv site of the matrix, revealed by the difference in the $G(r)$ functions of a pure argon matrix with that obtained for $\mathrm{Na}$ in a sv site. Expansion of the lattice is seen in the first, third, and fourth nearest-neighbor shells but not in the second surrounding sphere. To accommodate the large spherical guest atom, the first surrounding sphere expands radially. In so doing the rare gas atoms move directly toward the atoms in sphere four, perturbing both layers in the process. The second sphere remains largely undisturbed because the atoms in this shell do not lie directly in the path of the atoms of the expanding first shell.

Radial distributions for $\mathrm{Na} / \mathrm{Kr}$ are presented in the lower panels. Upon examination of both diagrams, it is clear that the results are similar to those reported above for $\mathrm{Na} / \mathrm{Ar}$. However, the extent of the distortion of the sv site is much less in $\mathrm{Kr}$ than in the case of Ar. As found in the $\mathrm{Na} / \mathrm{Ar}$ system, disruption of the lattice with a large guest in a sv site does not extend beyond the fourth sphere atoms.

A larger hv site is created with the removal of six rare gas atoms surrounding an octahedral interstitial site. The radial distribution in $\mathrm{Ar}$ when a $\mathrm{Na}$ atom occupies such a site is presented on the upper right in Figure 7. Nearest neighbors are at $4.45 \AA$ and next nearest neighbors at $5.85 \AA$, whereas the corresponding values in a pure Ar lattice are 4.60 and $5.94 \AA$, respectively. Thus the argon cage is slightly contracted around the sodium atom in a hv site. As shown in Figure 7, a similar contraction of the cage is obtained with $\mathrm{Na}$ in a hv site of solid krypton. In both cases, peaks are broad, due to the large amplitude motion of the sodium in this large site.

One can notice minor variations in the radial distributions obtained in this study compared to previous calculations described in ref 9.

V.B. Simulated Depositions. Simulating the sample sprayon depositions under the conditions described in part III.B revealed the difficulty in obtaining the sodium atom embedded in solid argon. Thus when argon atoms reach the surface, they more readily attach to other argon atoms than to $\mathrm{Na}$, with the effect that the sodium atom remains floating on the top of the surface. This behavior arises from the stronger binding energy of $\operatorname{Ar}_{2}\left(D_{\mathrm{e}}=99.2 \mathrm{~cm}^{-1}\right)$ compared with $\mathrm{Na} \cdot \operatorname{Ar}\left(41.5 \mathrm{~cm}^{-1}\right)$ and is a result entirely consistent with matrix experiments. Thus the $\mathrm{Na}_{2} / \mathrm{Na}$ ratio is greater in $\mathrm{Ar}$ than in the heavier rare gas matrices, because during deposition, the $\mathrm{Na}$ atom spends more time on the surface where atomic diffusion is fast, increasing the probability of forming sodium dimer. To be able to stabilize the monomer inside the lattice, the mass of the $\mathrm{Na}$ atom was increased from 23 to $40 \mathrm{amu}$. With the same mass as Ar, the sodium atom has a larger inertia while the $\mathrm{Ar}-\mathrm{Na}$ interactions (and the physical behavior) remain the same. The deposition temperature was varied between 5 and $25 \mathrm{~K}$. However, simulations undertaken above $20 \mathrm{~K}$ consistently failed to trap the $\mathrm{Na}$ atom.

Approximately one hundred simulated depositions were calculated for Na/Ar. Typically, 600 argon atoms are required to ensure that the $\mathrm{Na}$ atom is trapped in the argon matrix. The calculated radial distributions, $G(r)$, reveal the site of isolation of $\mathrm{Na}$ in $\operatorname{argon}$ to be a tv. The result is identical to that found for the $\mathrm{Na}$ atom in a model tv site illustrated in upper left panel of Figure 7. This was the only site ever found in the simulated depositions of $\mathrm{Na} / \mathrm{Ar}$.

Approximately ninety simulated depositions were completed for $\mathrm{Na} / \mathrm{Kr}$ using $\mathrm{MD}$ calculations. In general 400 atoms were required to ensure the $\mathrm{Na}$ atom was embedded in the $\mathrm{Kr}$ lattice. The temperature was varied between 5 and $45 \mathrm{~K}$; however, depositions above $35 \mathrm{~K}$ failed to trap the $\mathrm{Na}$ atom. Variation of the distance between the incoming atoms was also applied in order to increase or decrease the speed of deposition. The radial distributions calculated for simulated depositions matched the $G(r)$ values presented in the left and center panels shown in Figure 7 for the model tv and model sv sites respectively. Results show that the $\mathrm{Na}$ atom becomes isolated slightly preferably $(56 \%)$ in a tv site.

V.C. Simulated Absorptions. The simulated absorption spectrum for a $\mathrm{Na}$ atom in a tv site in a solid Ar matrix is compared in Figure 8 with the experimental spectra. 3-Fold splitting is clearly present in all three experimental spectra-a feature that is successfully reproduced in the simulated tv spectrum. However it occurs considerably to lower energy than the thermally stable blue site. Simulated absorption spectra from $\mathrm{sv}$ and hv sites are also reported in Figure 8 for comparison. It is evident that all three simulated spectra are energetically very close but match the red site only. Similar mismatches were obtained in the studies of Boatz and Fajardo, ${ }^{9}$ indicating that the use of the most accurate pair potentials seems only to provide a minor improvement. The simulated absorption spectra of $\mathrm{Na} /$ $\mathrm{Kr}$ are presented in Figure 9 in comparison with experimental results. The spectra simulated for the three sites are once again very close to each other and are located in the red part of the experimental profile. Considering that the blue band in the $\mathrm{Na}$ / 

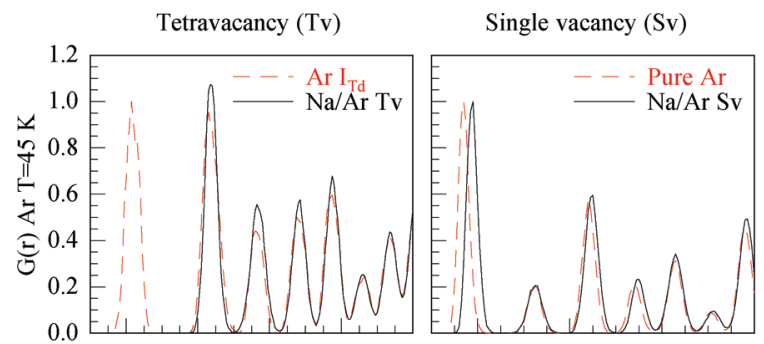

Hexavacancy (Hv)
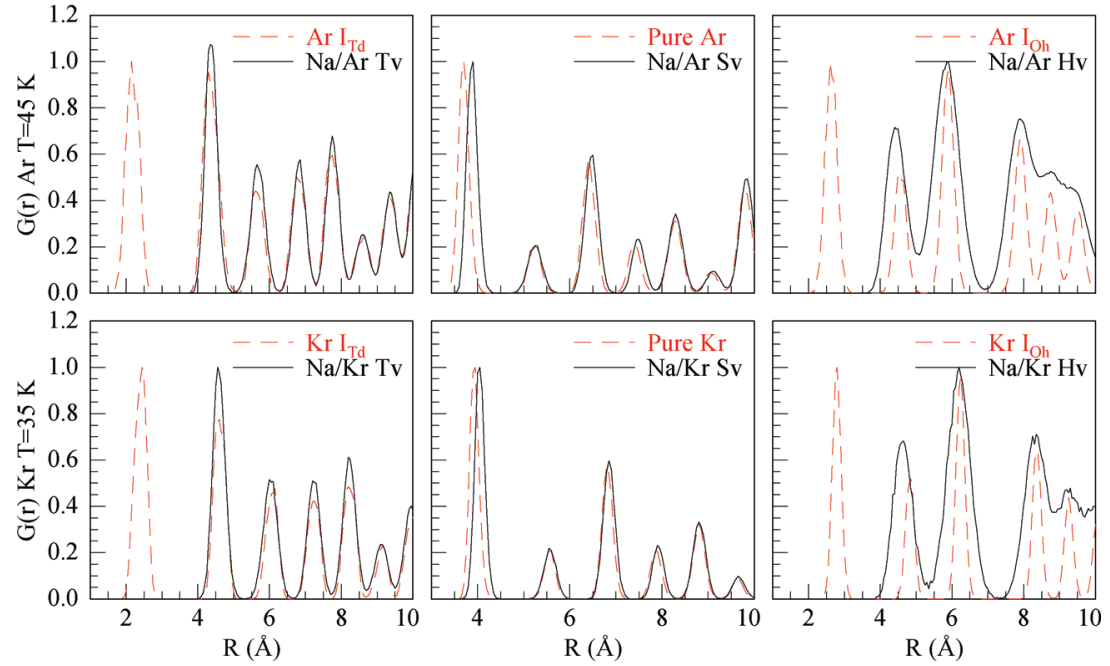

Figure 7. The top left panel illustrates the extent of the overlap of the radial distribution functions $[G(r)]$ of atomic Na placed in a tv site of an Ar matrix and the $G(r)$ function of a pure argon lattice. The top center and right panels present the same comparison but with Na in a sv site and in a hv site. The lower panels show the comparisons for $\mathrm{Na} / \mathrm{Kr}$. Comparison of the radial distribution of $\mathrm{Na}$ in a model tv site of an Ar cube with that found in a simulated deposition using the MD calculations (described in the text) reveals this is the only site formed in Ar. In contrast both sv and tv sites of $\mathrm{Kr}$ are found in the simulated $\mathrm{Na} / \mathrm{Kr}$ depositions. Thus these comparisons reveal tv site occupancy in Ar with simultaneous tv and sv sites present in $\mathrm{Kr}$ matrices. All the results are obtained with an effective temperature of $45 \mathrm{~K}$ in $\mathrm{Ar}$ and $35 \mathrm{~K}$ in $\mathrm{Kr}$ (see text).

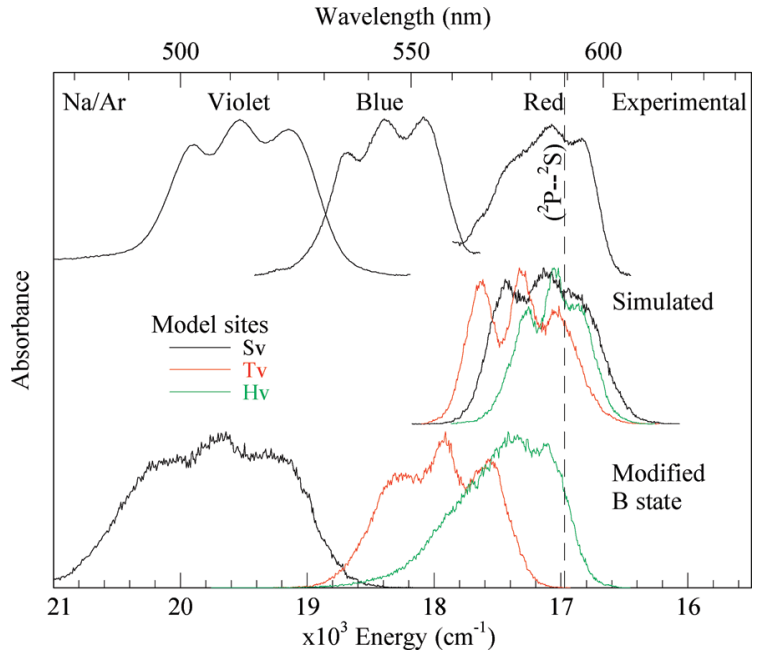

Figure 8. A comparison of the recorded absorption (excitation) spectra in $\mathrm{Na} / \mathrm{Ar}$ with the simulated absorptions for sv, tv, and hv site occupancy. The center panel shows the results obtained with the "exact" pair potentials. The bottom panel shows the effect of changing only the repulsive $\mathrm{B}$ state potential as shown by the dashed trace in Figure 1.

$\mathrm{Kr}$ experimental spectrum corresponds to the most stable site, all the simulated spectra are red-shifted from it. The agreement with experimental data is better than in the case of argon, but the assignment to tv or sv sites, found from simulations of deposition, cannot be made because of the vicinity of both simulated spectra.

The pair potentials used in these simulations are specified in Table 2 and are especially well fitted on the experimental gas phase data from 3.4 to $15 \AA$-the most important distance range for the atomic interactions occurring on the ground state surface in the solid. The absorption results presented in Figures 8 and 9 raise questions about the reliability of spectra simulated in the condensed phase with atomic pair interactions. To test the dependence of spectra on the pair potentials used, we have slightly modified these pair potentials. The bottom portion of Figure 8 presents spectra simulated for the three sites in argon

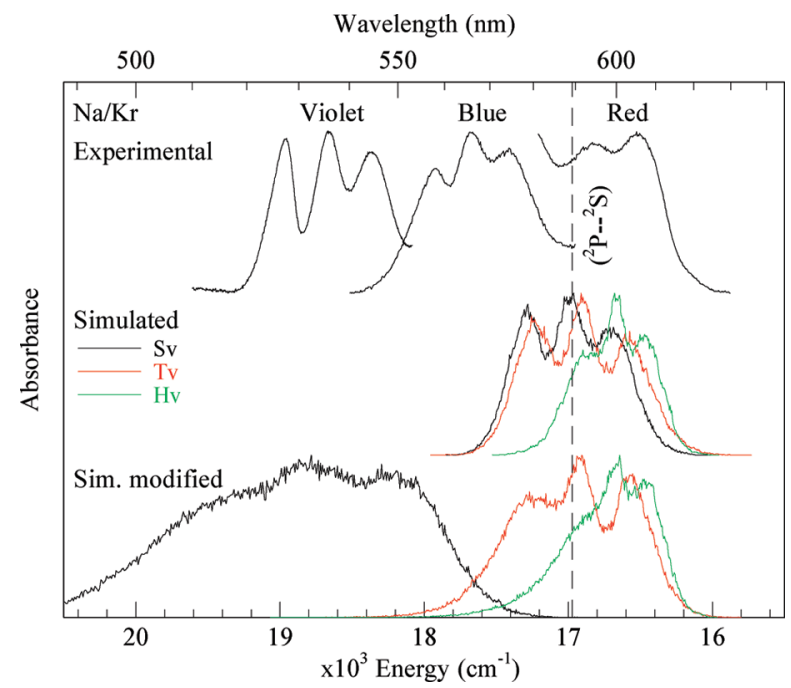

Figure 9. The same comparison presented in the previous figure but for the $\mathrm{Na} / \mathrm{Kr}$ system. Once again only the repulsive B state potential, shown the dashed trace on the right in Figure 1, was used to produce the results shown in the bottom panel.

with $\mathrm{Na}-\mathrm{Ar}$ pair potentials modified only in the B state. We chose to restrict modification to the $\mathrm{B}$ state, because of the limited experimental data that exists in the quasi-purely repulsive region of this state. On the other hand, since the $\mathrm{B}$ state presents the most repulsive potential in the $\mathrm{Na}-\mathrm{RG}$ distance range explored in the matrix, one can expect the most pronounced effect of the solid to be on this state. The modification of the B state, corresponding to an increase of the repulsive part at short distances and a weakening of the attractive part around $7 \AA$ (shown in the inset of Figure 1), induces differential blue shifts of the three sites and changes in the splitting patterns and widths of the absorption profiles. As shown in Figure 8, the absorption spectrum of the tv site matches the experimental blue site and the three components of the Jahn-Teller structure reproduce the observed behavior very well. One can notice the large blue shift of the sv spectrum, which based on the shape and the spectral location could be assigned to the "violet" experimental 

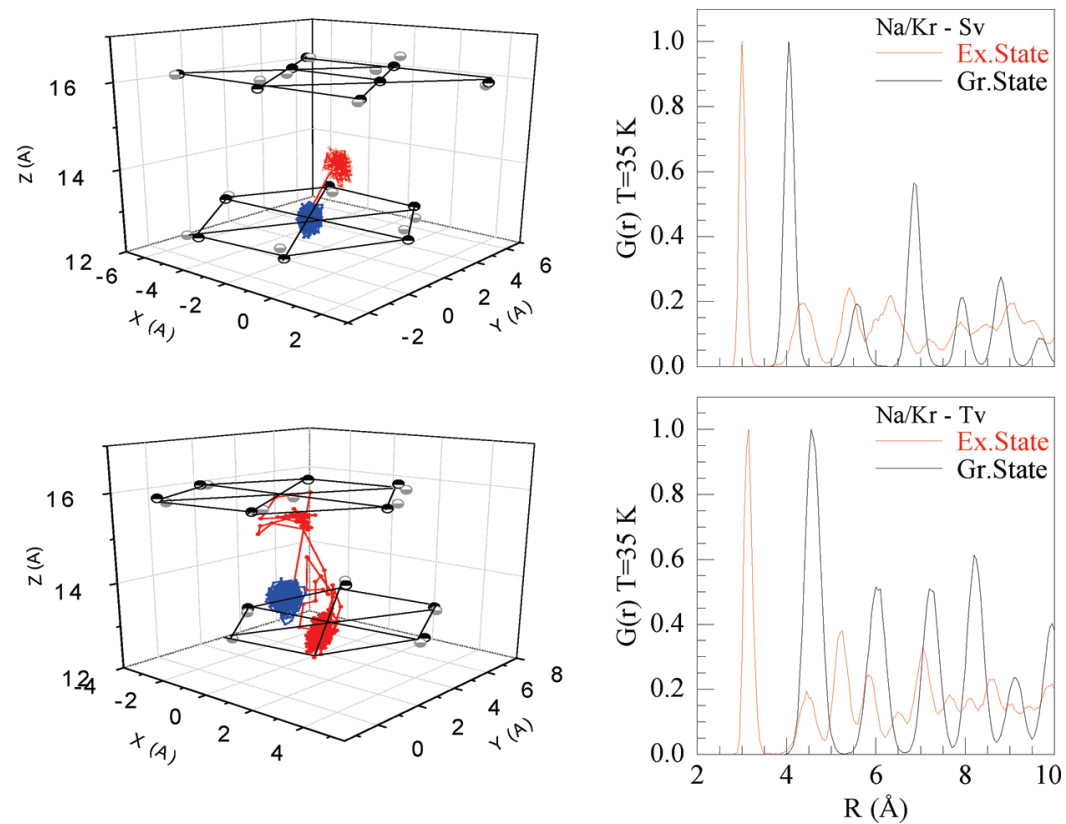

Figure 10. A trajectory for ground state atomic $\mathrm{Na}$ in a sv [tv] site of $\mathrm{Kr}$ is shown in blue at in the upper [lower] left-hand panel. Its movement from this initial position, when promoted to the excited state, is shown by the red line. The time scale of trajectories is $1 \mathrm{~ns}$, allowing the stabilization of $\mathrm{Na}$ in a new position in the case of the excited state. Snapshots of the positions of $\mathrm{Kr}$ atoms in the neighborhood are shown in black (initial positions of the red trajectory) and gray (final positions of the red trajectory) circles. Black lines are added to highlight equilateral triangles of (111) planes of the fcc lattice. In the panels on the right-hand side, a comparison of the radial distribution functions calculated for the sites occupied by $\mathrm{Na}$ atoms in the ground (black) and excited (red) states corresponding to the reported trajectories is made: upper, sv site; lower, tv site.

band. Using the modified B state potential the simulated absorption energies of the tv and sv sites are greater than the red experimental site. However, when this potential is used to simulate the hv site absorption, the resemblance with the red site is striking especially in relation to the complex asymmetric band-shape. The results of a similar modification of the B state (see dashed lines in Figure 1) in $\mathrm{NaKr}$ are shown on the bottom panel in Figure 9.

In the absence of a physical meaning of the changes in the affected B state pair potentials, we have not attempted to fit exactly the experimental spectrum. Nonetheless, this attempt shows that small modifications of the pair potentials can result in large energy shifts in the spectra and change very subtle details of their band profiles. While such a spectral dependence was expected, the effect of the B state potential was found negligible on the structure of small $\mathrm{Na}\left(3^{2} \mathrm{P}\right) \mathrm{Ar}_{n}$ clusters. ${ }^{41}$ This result has to be mentioned for the following description of dynamics in the excited state (see next part).

V.D. Simulated Emission. As described in "Theoretical Methods", the emission spectra are calculated from an equilibrium configuration on the excited state surface. To obtain this configuration, an investigation of the motion of the atom on the excited state potential energy surface was carried out before simulating emission spectra. In these calculations, the pairpotentials utilized reproduce very well Zimmermann's gas phase experimental potentials [see refs 22-25], even at the short distances explored by the excited sodium atom. Simulated emissions calculations were conducted for sv, tv, and hv sites. In all cases, a strong rearrangement of the lattice occurs in the neighborhood of the excited sodium atom.

Figure 10 presents examples of trajectories of the sodium atom in the excited state from initial tv (lower panel) and sv (upper panel) sites in krypton. Verification of these movements can be made when the trajectory of the ground state atom (shown in blue) is compared with that of the atom on the excited potential energy surface (shown in red). In the right-hand side of the same figure, the $G(r)$ functions of the excited sodium stabilized in the matrix in the corresponding final positions are presented in red and compared with $G(r)$ in the corresponding initial positions, shown in black. Inspection of the left panel in Figure 10 illustrates how the atom moves from the ground state single-vacancy or tetravacancy site to a smaller space in the excited state. At $t=0$ it can be seen that the atom begins in the same position as the ground state; however, when it is subjected to the new forces of the excited state potential energy surface (PES), it moves a large distance from its original position. The movement of the $\mathrm{Na}$ atom is also illustrated in the right-hand panel of Figure 10 by the shift of the first peak toward shorter equilibrium diatomic distance. The $G(r)$ calculation reveals that the shortest radial distance between the krypton atoms and the metal atom is $3.00 \AA$ for initial sv site occupancy and $3.15 \AA$ from the tv site. These distances are very close to the size of the $\mathrm{I}_{\mathrm{Oh}}$ site in $\mathrm{Kr}$, given in Table 1 as $2.82 \AA$. Thus in the excited state, the atom has moved toward an adjacent octahedral interstitial site irrespective of the site initially occupied in the ground state.

From all the trajectories explored in this study, including sv, tv, and hv initial sites in $\mathrm{Ar}$ and $\mathrm{Kr}$, a first peak around $3 \AA$ is obtained in the calculated $G(r)$ in the excited state. In all cases, it corresponds to four nearest neighbors at a better defined distance from $\mathrm{Na}$ than for any other rare gas atom in the lattice, as shown by the respective bandwidths of the $G(r)$ plots. This behavior can be understood when the short equilibrium distances $\left(2.94 / 3.03 \AA^{23}\right)$ of the strongly bound $\mathrm{A}^{2} \Pi$ state of the diatomics $\mathrm{Na} \cdot \mathrm{Ar} / \mathrm{Na} \cdot \mathrm{Kr}$ are considered. This state in $\mathrm{Ar}(\mathrm{Kr})$ has a binding energy of $581(795) \mathrm{cm}^{-1}$ in contrast to the weakly bound 41.5 (68.4) $\mathrm{cm}^{-1}$ ground $\mathrm{X}^{2} \Sigma$ state with a bond length of 5.01 (4.92) $\AA{ }^{23}$

For the $\mathrm{Na}$ atom occupying hv, tv, or sv sites, in both $\mathrm{Ar}$ and $\mathrm{Kr}$ lattices, a motion occurs in the excited state corresponding to a decrease of few $\mathrm{RG}-\mathrm{Na}$ distances in order to minimize the energy of the $\Pi$ interactions. These short distances involve 


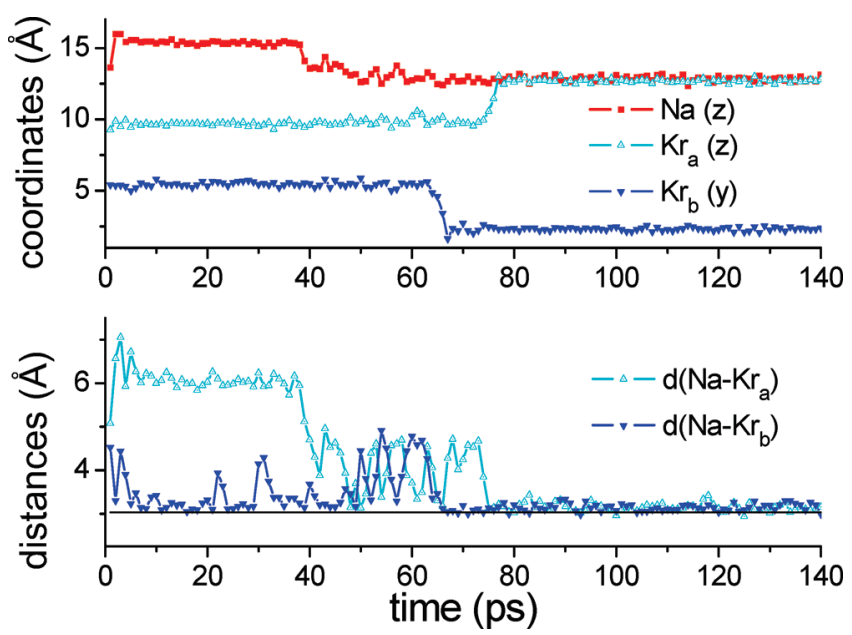

Figure 11. Time dependence of coordinates of $\mathrm{Na}$ and the two moving $\mathrm{Kr}$ atoms, labeled $\mathrm{Kr}_{\mathrm{a}}$ (light blue) and $\mathrm{Kr}_{\mathrm{b}}$ (dark blue) [upper panel] and corresponding $\mathrm{Na}-\mathrm{Kr}_{\mathrm{a}, \mathrm{b}}$ distances [lower panel] during the first $140 \mathrm{ps}$ of the trajectory reported in the left lower panel of Figure 10. $\mathrm{Na}$ is initially in a tv site, its movement following excitation, reported in Figure 10, involves mainly its $z$ component reported in red in the upper panel. The $z$ and $y$ components are chosen to represent the movement of $\mathrm{Kr}_{\mathrm{a}}$ and $\mathrm{Kr}_{\mathrm{b}}$, respectively, in the same plot. The successive move of $\mathrm{Kr}_{\mathrm{b}}$ and second $\mathrm{Kr}_{\mathrm{a}}$ following the motion of $\mathrm{Na}$ is clearly observed. These $\mathrm{Kr}$ movements represent a strong decrease of corresponding $\mathrm{Na}-\mathrm{Kr}$ distances (lower panel) showing a stabilization around $3 \AA$ for both distances, very close to the equilibrium distance of $\mathrm{Na} \cdot \mathrm{Kr}$ complexes in the A state, reported in this plot as the black horizontal line.

mainly four RG atoms. Thus, the motion of the excited sodium atom appears to be dominated by $\Pi$ interactions, without a great influence of the repulsive $\Sigma$ interaction. Actually, because of the vacancies created at the initial position of the $\mathrm{Na}$ atom, the $\Sigma$ interactions occur with RG atoms at longer distances, as shown from the $G(r)$ results. In agreement with this remark, very similar results have been obtained when the highly repulsive modified $\mathrm{B}$ state is taken into account. This result reinforces the validity of our simple approach which neglects the modification of the B $\Sigma$ interaction by the RG lattice. It is also consistent with previous calculations on $\mathrm{Na}^{*} \mathrm{Ar}_{n}$ clusters. ${ }^{41}$

In these conditions, emission occurs from a very disturbed site compared to the site initially occupied in the ground state. The distortion from a well-ordered site can be evaluated by a calculation of $G(r)$ at the equilibrium position on the lowest excited energy potential surface. With the exception of a welldefined peak corresponding to $\mathrm{Ar}$ or $\mathrm{Kr}$ atoms adjacent to $\mathrm{Na}$, in a position very close to $\mathrm{I}_{\mathrm{Oh}}$ sites, all the remaining peaks are broad and numerous. When coming from a sv site, the motion toward an $\mathrm{I}_{\mathrm{Oh}}$ site leaves only one vacancy. In the example of Figure 10, it leads to a direct trajectory. When coming from a larger site, the motion can occur toward less than four rare gas atoms because of the vacancies, so that the motion of rare gas atoms from one site to an empty one is possible. As a result, there is no longer any order in the neighborhood of the excited sodium. In Figure 10, two $\mathrm{Kr}$ atoms migrate in sequence after the $\mathrm{Na}$ atom has moved. Figure 11 presents the time dependence of these movements with the first $\mathrm{Kr}$ atom, shown in dark blue, and a second one in light blue. In this example, the trajectory was not direct, the excited sodium explores different geometries with close $\mathrm{Kr}$ atoms. It begins its stabilization very close to two $\mathrm{Kr}(\sim 40 \mathrm{ps})$ atoms, one more $\mathrm{Kr}$ is attracted near $\mathrm{Na}(\sim 65$ ps, dark blue) followed by a second one ( $\sim 75 \mathrm{ps}$, light blue), leading to a more compact site, involving four very close neighbors. One can notice a great similarity in $G(r)$ obtained from tv and hv sites, suggesting similar rearrangements of rare gas atoms in both cases, slightly different from the sv case, as shown in Figure 10. Moreover, these radial distributions exhibit the same features in $\mathrm{Ar}$ and in $\mathrm{Kr}$.

Emission spectra of $\mathrm{Na} / \mathrm{Ar}$ and $\mathrm{Na} / \mathrm{Kr}$ for the initial hv, tv, and sv sites are calculated from the excited equilibrium position. The emission spectra all contain one band, strongly red-shifted from the absorption profile. In argon, the maximum emission wavelengths of the simulated spectra are 939, 926, and $942 \mathrm{~nm}$ for the sv, tv, and hv sites, respectively. While the profiles and bandwidths are quite well reproduced, the spectral positions are not at all in agreement with experimental data. Experimentally, the red and blue site emissions in $\mathrm{Na} / \mathrm{Ar}$ are centered at 698 and $679 \mathrm{~nm}$, respectively (Table 3). Shifts between experimental emissions and simulated bands in $\mathrm{Kr}$ are also very important, but less than in Ar. These discrepancies are perhaps not so surprising when it is considered that emission occurs from lattice configurations with few short $\mathrm{RG}-\mathrm{Na}$ distances for which the ground state potential is very repulsive, and where a small change in positions is followed by big changes in energy.

The agreement in absorption between experimental spectra and simulation was not good and seems to be worse in emission. Taking account of this disagreement and the fact that the sites from which emission occurs presents some disorder, we have not performed many attempts in the calculation of emission spectra coming from different trajectories in the excited state. Nevertheless, some calculations have been performed with the modified B state in Ar, and they do lead to a blue shift in the simulated spectra but only slightly. The discrepancy with experiments is still huge even if the emission from the tv site, giving the largest energy, is now calculated around $870 \mathrm{~nm}$. Evidently, the lack of knowledge of the B state, especially its repulsive part, cannot explain the very poor agreement with experiments.

However, in agreement with experiments, the Stokes' shift is huge, explained by the very different equilibrium geometries in the ground state and in the excited state. Moreover, simulated emission spectra from sv, tv, or hv sites are very close, in simulations and experiments, because, in all the sites, emission is coming from similar positions involving a few very short $\mathrm{Na}-\mathrm{RG}$ distances.

\section{Discussion}

VI.A. Site Assignments. VI.A.1. Stable Sites. The polarizability plot presented in Figure 6 suggests a correlation between the violet sites in $\mathrm{Ar}$ and $\mathrm{Kr}$ and the blue site in $\mathrm{Xe}$. The two red sites in $\mathrm{Ar}$ and $\mathrm{Kr}$ do not have a corresponding band in Xe. Experimentally, the most thermally stable sites are the so-called "blue" sites. Simulated MD depositions indicate that the only site of isolation of atomic sodium in solid argon is a tv site, whereas tv and sv sites are obtained in Kr. As this method favors the formation of the most stable sites we accordingly propose tetravacancy as the site occupancy for the blue site in Ar. On the basis of the predominant trapping site obtained from MD simulations, but more especially the experimental correlations between $\mathrm{Ar}$ and $\mathrm{Kr}$, we propose the same assignment for the blue site in $\mathrm{Kr}$.

This assignment is also in agreement with the recent theoretical studies performed by Gervais and co-workers. ${ }^{7,42}$ In a previous work ${ }^{7}$ based on a time dependent density functional theory, it was concluded from energetic calculations that less destabilization of the Ar lattice occurs for $\mathrm{Na}$ atoms in a tv site than a sv site. Nevertheless, as in the present DIM simulations, estimations of optical absorption frequencies in both cases were 
very similar. Gervais's group has recently conducted ab initio calculations $^{42}$ on the same system giving more accurate spectral results and leading to the same site assignment. This assignment will be taken as a first statement in the following discussion.

VI.A.2. Unstable Sites. The red site in $\mathrm{Na} / \mathrm{Ar}$ has been assigned in previous ${ }^{2}$ works either to a site close to a lattice defect or to a divacancy site. A divacancy site has a very distinct site symmetry in which the three $3 p$ orbitals become nonequivalent, with one distinct orientation along the $z$-axis (diatomic internuclear axis) of the cylindrical cavity and two equivalent orientations perpendicular to this. Simulation of the absorption spectrum of such a site in a "model" RG cube gives two very distinct parts, one corresponding to absorption of the $3 \mathrm{p}_{z}$ component, and the other corresponding to the $3 \mathrm{p}_{x}$ and $3 \mathrm{p}_{y}$. From the experimental work carried out on $\mathrm{Na} / \mathrm{Ar}$, the red site exhibits 3-fold splitting in absorption; it was observed that the red site in $\mathrm{Kr}$ exhibits this splitting also. Thus the red site does not arise from a divacancy. From the observed shape it must be of cubic symmetry in both matrices. In a first approach, sv sites should be good candidates. They show the right symmetry, they were obtained in the simulation of deposition in $\mathrm{Kr}$, and simulated absorption profiles are close to the red absorption bands. The last point cannot however be presented as a supporting argument, because of the lack of spectral agreement in tv sites. Moreover, in the absence of strong theoretical arguments, one would expect a blue shift in absorption from tv to more cramped sites, as obtained with the use of the modified B state. Thus, further theoretical investigations were undertaken to answer this question. In this regard hcp structures were built instead of the fcc lattice but no differences were observed in the simulated absorption spectra for tv and sv sites. Simulations with the $\mathrm{Na}$ atom in an octahedral interstitial site $\left(\mathrm{Io}_{\mathrm{h}}\right)$ and a hv site of the fcc structure have also been carried out. As expected, $\mathrm{Io}_{\mathrm{h}}$ sites were easily eliminated as possible candidates. When the $\mathrm{Na}$ atom is placed in an $\mathrm{Io}_{\mathrm{h}}$ site in the krypton matrix, it migrates at all temperatures to a single-vacancy site. Thus the same calculation was not carried out in the Ar matrix but the red site is not arising from octahedral interstitial site occupancy. Such a $\mathrm{Io}_{\mathrm{h}}$ site was also found unstable in $\mathrm{Ar}$ in the theoretical investigations of Ahokas et al. ${ }^{6}$

In contrast to $\mathrm{Io}_{\mathrm{h}}$, hv sites could correspond to "red" sites. The thermal stability of such sites is very difficult to verify with MD simulations, because only a few nanoseconds can be reached in calculations, whereas modifications of the lattice structure requires much longer times. During a few ns, hv sites are found stable in simulations. In this site, the sodium atom is not restrained, a characteristic that is particularly obvious from the $G(r)$ results shown on the right in Figure 7. Because of the weakness of interactions with the rare gas neighborhood, the absorption frequency should not shift significantly from the gas phase line, as indeed is the case for the "red" sites. In agreement, modification of the B state potentials do not greatly affect the spectral position of simulated absorption in hv sites, with positions only slightly shifted from the gas phase value. As shown in Figures 8 and 9, the hv sites shift slightly to the blue in $\mathrm{Ar}$ and to the red in $\mathrm{Kr}$; both bands match the experimental "red" site profiles very well. Considering this behavior, it is clear that "violet" sites cannot be assigned to such large sites. We conclude that "red" sites correspond to large holes with cubic symmetry, that is, hv sites.

In fact, the present study provides new arguments which support assignment of the "violet" sites to sv configurations. Fajardo and co-workers had deduced from their studies that this "new" site was a tight site ${ }^{3,9}$ as the sv site for Na in Ar certainly is. Later, Ahokas et al. ${ }^{6}$ assigned the new site obtained in samples prepared by laser ablation to sv sites. Such cramped sites could appear using this sample preparation technique through an initial trapping of ionic sodium. ${ }^{8}$ In the present experimental study, the same site is obtained through a strong resonant laser irradiation of the sample. A plausible explanation of this laser-induced behavior is the following. From MD simulation in the excited state, we observed a significant rearrangement of the rare gas atoms around the excited sodium, due to the strongly attractive interactions occurring on the $A^{2} \Pi$ state. Such movements of rare gas atoms toward the guest $\mathrm{Na}$ atom will create sv sites from larger vacancies like tv or hv sites. Thus, we propose the assignment of "violet" sites to sv sites.

VI.B. Spectra and Pair-Potentials. As already mentioned, the absorption and emission spectra simulated with the methods outlined in section III and using accurate pair potentials derived from gas phase spectroscopy, give poor agreement with the experimental observations in matrices. The mismatch should come mainly from the description of the excited states since the ground state involves only weak van der Waals interactions between close-shell spherical species. The exception involves calculations of emission spectra coming from the very short distances present in geometries close to $\mathrm{Io}_{\mathrm{h}}$ sites. For these reasons the calculated emission frequencies are especially doubtful. Within a pair potential description, the interactions between the excited sodium and a rare gas atom are only of two kinds, viz, the attractive $\Pi$ and repulsive $\Sigma$ interactions, described by A and B state pair potentials, respectively. The attractive part is especially well described in the range of the explored $\mathrm{Na}-\mathrm{RG}$ distances from the very precise experimental data ${ }^{22-25}$ that now exists on the $\mathrm{Na} \cdot \mathrm{RG}$ diatomics. These data show that the A state potential wells are much deeper than the ground state, but for $\mathrm{Na} \cdot \mathrm{Ar}^{23}$ and $\mathrm{Na} \cdot \mathrm{Kr}^{24}$ they do not exceed $1000 \mathrm{~cm}^{-1}$.

Thus, as a first attempt to improve the DIM method, one can only modify the B potential in order to take into account solid state effects and build an "effective" potential. Such an approach was successfully used by G. Rojas-Lorenzo et al. ${ }^{43}$ on NO-Ne pair potentials to reproduce the dynamics of the lattice following excitation of NO to its Rydberg state in solid neon. Without any further theoretical support, we have attempted a simple empirical modification of this B state, involving a more pronounced repulsive part, in order to blue-shift the absorption spectra simulated with the previous calculations, and thereby obtain better agreement with experiments. The resulting spectra in $\mathrm{Ar}$ presented on the bottom in Figure 8 are entirely in agreement with the previously proposed site assignments (part A). In sites of smaller volume, the repulsive region of the excited state dominates the interactions experienced by the guest atom. As a result, the difference in energy between the ground and excited states is greater than that of a larger site and the atom absorbs at higher energies. Beside the frequency agreements, even the shapes of absorption profiles are quite well reproduced. The agreement in $\mathrm{Kr}$ is not as good as in Ar, but confirms the assignments of "violet", "blue" and "red" sites to sv, tv, and hv sites, respectively.

We have not tried to reach the best possible agreement using a modified B potential. This attempt was performed essentially to highlight the sensitivity of the results on the pair potentials selected in the DIM method. This problem will be discussed in a next paper devoted to $\mathrm{Na} / \mathrm{Xe}$. In $\mathrm{Xe}$, the repulsive part of the pair potentials is less explored in sv, tv, or hv sites than in $\mathrm{Ar}$ or $\mathrm{Kr}$, so the results from the same simulation method are 
expected to be more accurate. Investigations and discussion of this last system will be the subject of the next paper. The charge distribution in $\mathrm{p}-\Sigma$ interaction should be particularly modified by the rare gas surrounding. Such behavior cannot be described within the formalism of the used DIM method, including only the A and B pair potentials. The small number of accessible parameters does not allow a full exploration of the phase space of the charge-separated system. More sophisticated theoretical approaches, ${ }^{16,17,42}$ including higher excited states, through ab initio methods, are necessary for a complete understanding of the spectral features.

VI.C. Simulation Dynamics. Taking the current site assignments into account, it is surprising that a sv site was found during simulated deposition in $\mathrm{Kr}$, whereas hv sites were not found in either this or solid Ar. These findings can be understood when it is considered that the simulated depositions begin on a regular fcc lattice so that the building of a compact lattice is favored. For this reason, the ejection of the sodium atom to the surface of the matrix during Ar deposition frequently occurred. The effect of small lattice defects cannot be taken into account in the simulations, with the effect that larger vacancies, which could arise from slight deviations from the perfect fcc order, are not produced. This is consistent with the experimental annealing effect, decreasing the amount of red sites. On the other hand, a very weak absorption band corresponding to the violet site is observed on deposition in Kr. While it is too weak to be sure of the assignment of a sv site in $\mathrm{Kr}$, the corresponding site is not present in Ar on deposition.

As already mentioned, dynamics on the excited state energy surfaces are mainly controlled by $\Pi$ interactions. That is why similar emission results are obtained with different B potentials. The confidence of the description of this interaction is high enough to conclude that large motions of $\mathrm{Na}$ atoms occur with excitation. The first motion for the sv site occurs in less than 1 ps and is not analyzed in the present study. On the basis of previous work by Gerber and co-workers ${ }^{44}$ on matrix isolated atomic fluorine, this is the time the excited $\mathrm{p}$ state $\mathrm{Na}$ atom requires to reorient itself and adapt to the new forces of the applied PES. After which the "aligned" atom moves toward rare gas atoms and becomes stabilized close to four RG where it remains for the duration of the calculation. The state $E_{3}$ corresponds to the $\mathrm{p}_{z}$ orbital occupied while the approximately isoenergetic $\mathrm{E}_{1}$ and $\mathrm{E}_{2}$ states pertain to the $\mathrm{p}_{x}$ and $\mathrm{p}_{y}$ orbitals. This motion of the excited state $\mathrm{Na}$ atom corresponds to what was labeled the "body" mode in earlier calculations conducted by $\mathrm{us}^{45}$ on a much smaller $\mathrm{Zn}(\mathrm{RG})_{18}$ system.

Quite similar behaviors were found for the sv, tv, and hv sites with the excited atom moving close to one of the surrounding $\mathrm{I}_{\mathrm{Oh}}$ sites. The radial distributions around the excited sodium are especially similar in tv and hv sites. This is thought to be the reason the emission energies of the hv, tv, and sv sites are so similar. However, the time scale of the dynamics is completely different in large sites to that found for the sv site. Thus, as shown in the example of Figure 10 in the tv site, extensive rearrangement (lower left panel in Figure 10) occurs in the lattice atoms surrounding the void created by the $\mathrm{Na}$ atom approaching an $\mathrm{I}_{\mathrm{Oh}}$ site. As shown in Figure 11, the $\mathrm{Na}$ atom immediately approaches a pair of RG atoms, this motion is followed sequentially by two other atoms until at $80 \mathrm{ps}$, the $\mathrm{Na}$ atom is surrounded by $4 \mathrm{Rg}$ atoms at a distance optimal for the $\mathrm{A}^{2} \Pi$ state interaction. This distance matches approximately the nearest neighbor distance of an $\mathrm{I}_{\mathrm{Oh}}$ site, indicated in Figure 11 by the horizontal line.

\section{Conclusions}

Simulated depositions have found only a tetravacancy site for sodium atoms in Ar while this site and a single vacancy site are found for Kr. The absorption spectra of these sites, calculated with the DIM method, using highly accurate gas phase pair potentials, only agree with the red site present in the absorption spectra. An empirical modification of the B state in $\mathrm{NaAr}$ gave very good agreement with the red, blue, and violet bands for the hv, tv, and sv sites, respectively. Taken in conjunction with polarizability plots of the matrix shifts and dynamical behavior, this result suggests the red site is a hexavacancy, the blue site is a tetravacancy, and the violet site is a single vacancy. While the modified B state gave excellent agreement with absorption they did not yield significant improvement with emission data. Thus it appears that the use of the DIM method using the most accurate gas phase pair potentials does not provide an adequate description of the excited state $\mathrm{Na}$ atom interaction in the rare gas matrices. The reason for this is currently not clear but in a metal atom with a small ionization potential, three (or multi-) body interactions which may arise from charge separation cannot be described adequately by the DIM method. It is hoped that ab initio calculations will reveal the role played by such excited state behavior.

Acknowledgment. Fruitful discussions with Dr. Benoit Gervais are kindly acknowledged. This work was supported in part by the Irish Government Embark Initiative (IRCSET) programme Grant. In addition, J.McC. gratefully acknowledges Prof. invitee positions at Université Paris Sud 11 (hosted by Dr. C. Crépin) which commenced this work and recently at Université Pierre et Marie Curie, Jussieu (hosted by Prof. L. Krim) which concluded this work.

\section{References and Notes}

(1) Balling, L. C.; Havey, M. D.; Dawson, J. F. J. Chem. Phys. 1978, 69,1670

(2) Balling, L. C.; Wright, J. J. J. Chem. Phys. 1984, 81, 675.

(3) Tam, S.; Fajardo, M. E. J. Chem. Phys. 1993, 99, 854.

(4) Rose, J.; Smith, D.; Williamson, B. E.; Schatz, P. N.; O'Brien, M. C. M. J. Phys. Chem. 1986, 90, 2608. 485 .

(5) Crépin-Gilbert, C.; Tramer, A. Int. Rev. Phys. Chem. 1999, 18,

(6) Ahokas, J.; Kiljunen, T.; Eloranta, J.; Kunttu, H. J. Chem. Phys. 2000, 112, 2420.

(7) Gervais, B.; Giglio, E.; Jacquet, E.; Ipatov, A.; Reinhard, P. G.; Suraud, E. J. Chem. Phys. 2004, 121, 8466.

(8) Silverman, D. C.; Fajardo, M. E. J. Chem. Phys. 1997, 106, 8964.

(9) Boatz, J. A.; Fajardo, M. E. J. Chem. Phys. 1994, 101, 3472.

(10) Gross, M.; Spiegelmann, F. J. Chem. Phys. 1998, 108, 4148.

(11) McCarty, M.; Robinson, G. W. Mol. Phys. 1959, 2, 415.

(12) Ruffolo, M. G.; Ossicini, S.; Forstmann, F. J. Chem. Phys. 1985, $82,3988$.

(13) Jahn, H. A.; Teller, E. Phys. Rev. 1936, 49, 874.

(14) Jahn, H. A.; Teller, E. Proc. R. Soc., A (London) 1937, 161, 220.

(15) Roncero, O.; Beswick, J.; Halberstadt, N. Dynamics of Polyatomic Van der Waals Complexes; Halberstadt, N., Janda, K., Eds.; Plenum Press: New York, 1990; pp 471.

(16) El Hadj Rhouma, M. B.; Ben Lakhdar, Z.; Berriche, H.; Spiegelman, F. J. Chem. Phys. 2006, 125, 084315.

(17) Langhoff, P. W. J. Phys. Chem. 1996, 100, 2974

(18) Collier, M. A.; McCaffrey, J. G. J. Chem. Phys. 2003, 119, 11878.

(19) Collier, M. A.; McCaffrey, J. G. J. Chem. Phys. 2005, 122, 054503.

(20) Barker, J. A. Rare Gas Solids; Academic Press: London, 1976; Vol. 1.

(21) Maitland, G. C.; Rigby, M.; Smith, E. B.; Wakeham, W. A. Intermolecular Forces: Their Origin and Determination; Oxford University Press: New York, 1987.

(22) Schwarzhans, D.; Zimmermann, D. Eur. Phys. J., D 2003, 22, 193.

(23) Schwarzhans, D. Ph.D. Dissertation. Technische Universitat, Berlin, Germany, 2000; http://edocs.tu-berlin.de/diss/2000/schwarzhans_dirk.htm. (24) Bruhl, R.; Kapetanakis, J.; Zimmermann, D. J. Chem. Phys. 1991, 94,5865 . 
(25) Zanger, E.; Schmatloch, V.; Zimmermann, D. J. Chem. Phys. 1988, $88,5396$.

(26) Tang, K. T.; Toennies, J. P. J. Chem. Phys. 1984, 80, 3726.

(27) Thakkar, A. J. J. Chem. Phys. 1975, 62, 1693.

(28) Ahlrichs, R.; Penco, R.; Scoles, G. Chem. Phys. 1977, 19, 119.

(29) Kerner, C.; Meyer, W., Private communication from F. Rebentrost, 2006.

(30) Kerner, C. Ph.D. Thesis. Universitat Kaiserslautern, Germany, 1996.

(31) Grosser, J.; Hoffmann, O.; Rebentrost, F. J. Phys. B 2000, 33, L577.

(32) Grosser, J.; Hoffmann, O.; Klose, S.; Rebentrost, F. Europhys. Lett. 1997, 39, 147.

(33) Crépin, C.; de Pujo, P.; Bouvier, B.; Brenner, V.; Millié, P. Chem. Phys. 2001, 272, 243.

(34) Berendsen, H. J. C.; Postma, J. P. M.; Vangunsteren, W. F.; Dinola, A.; Haak, J. R. J. Chem. Phys. 1984, 81, 3684.

(35) Verlet, L. Phys. Rev. 1967, 159, 98

(36) Visticot, J. P.; de Pujo, P.; Mestdagh, J. M.; Lallement, A.; Berlande, J.; Sublemontier, O.; Meynadier, P.; Cuvellier, J. J. Chem. Phys. 1994, 100, 158
(37) Bergsma, J. P.; Berens, P. H.; Wilson, K. R.; Fredkin, D. R.; Heller, E. J. J. Phys. Chem. 1984, 88,612 .

(38) Tully, J. C. J. Chem. Phys. 1990, 93, 1061

(39) Rojas-Lorenzo, G.; Rubayo-Soneira, J.; Alberti, S. F.; Chergui, M. J. Phys. Chem. A 2003, 107, 8225.

(40) Fulton, R. F. J. Chem. Phys. 1974, 61, 4141.

(41) Tutein, A. B.; Mayne, H. R. J. Chem. Phys. 1998, 108, 308

(42) Douady, J. Ph.D. Thesis. Université de Caen, 2007; http:// tel.archives-ouvertes.fr/tel-00264921/fr/.

(43) Rojas-Lorenzo, G.; Rubayo-Soneira, J.; Vigliotti, F.; Chergui, M. Phys. Rev. B 2003, 67, 115119

(44) Krylov, A. I.; Gerber, R. B. Chem. Phys. Lett. 1994, 231, 395.

(45) McCaffrey, J. G.; Kerins, P. N. J. Chem. Phys. 1997, 106, 7885.

(46) http://physics.nist.gov/cgi-bin/AtData/(accessed May 28, 2009).

(47) Hallam, H. E.; Schrimshaw, G. F. Vibrational Spectroscopy of Trapped Species; Hallam, H. E., Ed.; Wiley: New York, 1973; pp 51.

JP905596A 